\title{
III-V Quantum-Dot Lasers Monolithically Grown on Silicon
}

\author{
Mengya Liao, Siming Chen*, Jae-Seong Park, Alwyn Seeds and Huiyun Liu* \\ Department of Electronic and Electrical Engineering, University College London, London, WCIE 7JE. UK
}

Email: siming.chen@ucl.ac.uk; huiyun.liu@ucl.ac.uk

\begin{abstract}
:
The monolithic growth of III-V semiconductor lasers on Si remains the 'holy grail' for full-scale deployment of Si photonics with reduced cost and added flexibility. Further, semiconductor lasers with active regions made from quantum dots (QDs) have shown superior device performance over conventional quantum well (QW) counterparts and offer new functionalities. There are other advantages of QDs for monolithic III-V-on-Si integration over QWs, such as QD devices being less sensitive to defects. It is, therefore, not surprising that the past decade has seen rapid progress in research on monolithic III-V QD lasers on Si, with a view to leveraging the benefits of QD gain region technology while benefitting from the economics of scale enabled by monolithic growth. This review has a special focus on O-band III-V QD lasers monolithically grown on Si for Si photonic optical interconnects, including Fabry-Perot lasers, distributed-feedback laser array, and micro-lasers. The successes and challenges for developing monolithic III-V lasers on Si are discussed.
\end{abstract}

Key words: Quantum dot, Semiconductor lasers, Monolithic Integration, Molecular Beam Epitaxy, Si Photonics.

\section{Introduction}

The beauty of Si photonics stems from its ability to combine Si microelectronics and photonics on the same chip that offer the tantalizing promise of cost-effectively fulfilling the ever-growing requirements on optical data transmission speed and bandwidth [1]. While, over the last few decades, the Si photonics community has made tremendous progress towards siliconizing individual photonic building blocks, including modulators [2-4], detectors [5-7], waveguides [8, 9], and multiplexers/demultiplexers [10][11], the absence of an integrated Si laser is a fundamental obstacle to widespread deployment. Si has an indirect bandgap that impedes efficient radiative recombination and thus blocks lasing [12]. Despite recent advances in group IV-based light emitters, such as $\mathrm{Si}$ Raman lasers [13, 12], germanium-on-Si lasers [14], GeSn-on-Si lasers [15], these lasers will not, in the foreseeable future, outperform their III-V counterparts. As a result, much effort has been directed towards hetero-integration of high-performance III-V lasers on Si substrates, both by hybrid waferbonding and by direct epitaxial growth. Despite the fact that the former approach constitutes the most mature and successful approach for creating III-V lasers on Si [16-20], the latter one, in the long term, remains the ultimate dream for cost-effective high-volume integration of photonic circuits [21-23].

Semiconductor lasers utilizing quantum dots (QDs) as the active region have been extensively investigated over the past decades. To date, III-V QD lasers have shown superior device performance over the conventional quantum well $(\mathrm{QW})$ lasers in terms of lower threshold and significantly improved temperature insensitivity [24, 25]. Recently, QD technology has gained considerable interest in monolithic III-V-on-Si integration due to its robust tolerance to defects which can be 
attributed to the effective carrier localization in the self-assembled nanostructures and hence reduced probability of interaction with defects [26, 27]. It is not surprising that QD lasers grown on Si have won an overwhelming victory over QW lasers grown on Si in many respects and demonstrated device performance and reliability that is comparable to lasers grown on native III-V substrates.

The combined strengths of QD active region techniques and monolithic III-V-on-Si integration technology are poised to revolutionize Si photonics. In this review article, we summarise recent achievements for O-band III-V InAs QD lasers epitaxially grown on Si substrates and describes how QD technology has opened up new avenues in monolithic III-V-on-Si integration technology, enabling efficient and reliable Si-based lasers for Si photonics. This review starts with the challenges associated with the monolithic integration of III-Vs on $\mathrm{Si}$ substrates in Section 2, followed by discussion of the advantages of QD over QW structures for use in monolithic integration in Section 3. Section 4 focuses on the $1.3 \mu \mathrm{m}$ InAs QDs lasers monolithically grown on Ge substrates, Ge-on-Si substrates, Si substrates with off-cut and on-axis Si (001) substrates. Section 5 describes monolithic QD distributed feedback laser arrays on $\mathrm{Si}$ substrates operating at $\mathrm{O}$ band. Before the Conclusion, Section 6 discusses $1.3 \mu \mathrm{m}$ QD micro-lasers monolithically grown on Si substrates.

\section{Challenges for monolithic integration}

The idea of monolithic integration of III-V materials on Si platform is, of course, not a new topic. First attempts to growth GaAs thin films on Si substrates started in the 1980s [28, 29] and continued in the 1990s with InP-based materials on $\mathrm{Si}$, especially at NTT in Japan [30, 31]. This approach, despite being investigated for decades, yielded little progress. This is because, unfortunately, monolithic III-V-on-Si integration poses critical issues stemming from the large material dissimilarity between III-V and Group IV materials, including polar III-Vs versus non-polar Si surfaces, large lattice mismatch and different thermal expansion coefficients [32-34].

The first issue, as seen in Figure 1(a), is the formation of antiphase boundaries (APBs) when growing polar III-Vs on nonpolar Si substrates. In practice, monoatomic steps exist on clean $\mathrm{Si}$ (001) surfaces [35], on which Si atoms form dimers in such a way that the dimers on one terrace are perpendicular to its neighboring one. This induces the formation of two domains as the III-V epitaxial proceeds with opposite sublattice allocation [36]. The contacting planes separating two domains consist of the same species, either III-III or V-V bonds, which are known as APBs. APBs are electrically charged planar defects, and act as non-radiative recombination centers and leakage paths for optoelectronic devices and electronic devices, respectively. The negative influence of APBs on optical properties of III-V materials is evidenced by photoluminescence (PL) quenching and spectral linewidth broadening [3738], while their impact on electronic devices is characterized by significantly reduced electron mobilities [38, 39].

The second issue, as seen from Figure 1(b), is related the formation of threading dislocations (TDs) in the III-V layers grown on Si. The lattice mismatch between GaAs (InP) and $\mathrm{Si}$ is about $4 \%(7.5 \%)$. This mismatch results in strain on the lattice of the epilayer and the strain energy is proportional to the deposition thickness. If the thickness of the epilayer grown exceeds the pseudomorphic critical point, the accumulated strain is energetically relaxed during epitaxial growth through the nucleation of dislocation half loops at the interface [36], creating, in general, two types of dislocations: misfit dislocations and TDs on the order of $10^{9}-10^{10} \mathrm{~cm}^{-2}$. The former, concentrated at the III-V/Si interface, enables the release of the strain energy, while the latter propagates into the III-V materials, acting as 
non-radiative recombination centers and eventually promoting defect diffusion along the dislocation line [40], hence reducing the quality of III-V materials as well as the device performance including operating characteristics and lifetime [34].

The last issue is related to the creation of thermal cracks, as seen from Figure 1(c), due to the difference in the coefficient of thermal expansion between III-V and group IV materials. This difference promotes the accumulation of thermal stress, which is then relieved by emerging thermal micro-cracks in the epilayer while the wafer was cooled down from the growth temperature to room temperature. The large difference in coefficient of thermal expansion limits the maximum achievable thickness of III-Vs which can be grown on $\mathrm{Si}$, and the typical critical thickness for the onset of crack formation in GaAs epilayers on $\mathrm{Si}$ is $\sim 7 \mu \mathrm{m}$ for a growth temperature of $\sim 575{ }^{\circ} \mathrm{C}$ [41].

\section{Advantages of $Q D$ for monolithic integration}

The idea of using heterostructures as the active region in lasers was first theoretically predicted by Kroemer in 1963 [42], but it was not until the demonstration of the first double heterostructure semiconductor lasers in 1970 [43], that the importance of carrier confinement to the active region was discovered. After that, the semiconductor laser community made tremendous progress in implementing quantum confined semiconductor lasers. At the first stage, very thin semiconductor layers, known as QW structures were utilized in the active region. Since the carriers in this structure are confined in one dimension, the thermal energy distribution, in comparison to bulk materials, was reduced due to the modifed density of states (DOSs), (see Figure 2 left and middle), which result in reduced threshold current and improved temperature stability. Now, QW lasers have become standard semiconductor lasers for many kinds of applications. Before QW lasers became typical semiconductor lasers, the novel idea of employing QDs for the active region was first theoretically proposed by Arakawa and Sakaki in 1982 [44]. Owing to an even greater modification of the DOSs, the carrier diffusion in the active region is significantly suppressed compared with its bulk and QW counterparts, since the carriers are strongly localised in the dots. Also, while there are certain distribution mechanisms existing, including homogenous and inhomogeneous distribution, which lead to broadening effect, the fundamental thermal distribution, when compared with bulk and QW active regions, as seen from Fig. 2 right, has been reduced considerably. It was therefore possible to realize a low threshold current density and high temperature performance laser [25].

However, turning those predictions into reality became possible only after the Stranski-Krastanow (SK) growth method to form self-assembled QDs came into use in the early 1990s [45]. Since then, academic research groups all over the world have been competing against each other to demonstrate QD lasers with dramatically reduced threshold current densities [46-52]. As shown in Figure 3, in a period of less than ten years, the performance of QD lasers (regarding threshold current density) has surpassed that of state-of-the-art QW lasers which have been long developed since 1980s [34]. It should be noted that since the device dimension, fabrication procedure and lasing emission for the lasers listed in Fig. 3 were different, comparing the threshold current has a level of an arbitrary process, the main purpose however is to provide a general ideal of the historical development of heterostructure semiconductor laser regarding the record threshold current density at the time of publication. Although QD lasers soon exhibit the predicted outstandingly low thresholds, these early devices suffered from the inhomogeneous broadening arising from QD size fluctuation [25], and also did not show the predicted good temperature stability mainly due to the thermally broadened hole distributions through closely spaced hole levels [53]. Due to the combination of improvements in size 
uniformity and dot density, especially, p-type modulation doping emerged that enabled QD lasers to offer good temperature stability. The key, at this point, is to counter the closely spaced energy levels by filling the excess holes [53, 54]. Now, QD Laser Inc. one of the world leading suppliers of QD lasers has delivered commercial products which are nearly temperature-insensitive up to $100{ }^{\circ} \mathrm{C}$ as shown in Figure 4 [55]. Moreover, the maximum operation temperature that can be achieved from their QD lasers is as high as $220{ }^{\circ} \mathrm{C}$ [25]. Also, QD lasers have demonstrated other important properties, including narrow spectrum linewidth [56], large modulation bandwidth [57, 58], long lifetime [25], and the possibility of making single photon sources [59]. In addition, it has been shown that the QD lasers have superior dynamic characteristics such as reduced sensitivity to optical feedback [60-62] and low relative intensity noise (RIN) [63-65]. In particular, it is important to achieve low RIN in semiconductor lasers, because the RIN increases the bit-error rate of optical signals and hence degrades the data transmission performance [66]. Accordingly, the RIN characteristics of QD lasers have been widely investigated [67, 68]. For example, it was reported that the InAs/GaAs QD lasers exhibited a RIN as low as $-160 \mathrm{~dB} / \mathrm{Hz}$ at frequencies up to $10 \mathrm{GHz}$, ascribed to the unique carrier dynamics offered by the QD gain media [63, 64].

In addition to the well-established features discussed above, very recently, III-V QD structures have obtained increasing attention due to the potential implementation of high-performance III-V lasers on $\mathrm{Si}$ substrates for Si photonics. One of the great benefits of QDs is that they are less sensitive to TDs than conventional bulk materials and QW structures, ascribed to carrier localization and hence reduced interaction with the TDs [69]. As shown in Figure 5(a), for conventional QW lasers, any TDs propagating through the QWs serve as a non-radiative recombination centre, resulting in an increased threshold and shortened operating lifetime. In contrast, for QD structures, one TD can only 'kill' one or a very limited number of dots, while still leaving the rest of dots intact and capable of providing sufficient optical gain to achieve lasing [27]. Moreover, it is well-known that the QD structures are able to bend the TDs and hence reduce the density of TDs [69-71]. As shown in Figure 5(b), the dislocation bending can occur beneath the QDs when a strain energy is released through the generation of the misfit dislocation under assumption that the shape of self-assembled QDs is pyramids and a TD propagates toward the bottom of a QD. Consequently, the bending of dislocation generated a segment of misfit dislocation gliding below the island [69]. This concept was observed by transmission electron microscopy (TEM) measurements, as shown in Figure 5(c). The TD was slightly bent around the QD array due to the strong strain field of the QDs (left side of Figure 5(c)), and even one of these QDs is able to help the TD bending toward the sample edge, rather than propagating perpendicularly to the surface (right side of Figure 5(c)) [72]. Therefore, even in the presence of high-density dislocations, QD lasers are able to provide superior reliability compared to QW or bulk devices $[25,36]$.

To further confirm this hypothesis, Liu et al from UCSB [73] made a direct comparison of QD lasers with QW lasers, all grown on $\mathrm{Si}$ substrates with similar dislocation densities. To make a fair comparison and separate other factors that may influence the device performance, all the techniques employed in their study, including the growth, processing, and measurement, were kept identical. Figures 6(a) and 6(b) compares the room temperature (RT) PL spectra of identical QD (QW) structures grown on Si and native GaAs substrates, respectively. While the integrated PL intensity of InAs QDs grown on Si only dropped roughly by $20 \%$, the integrated PL intensity of $\operatorname{In}_{0.2} \mathrm{Ga}_{0.8} \mathrm{As}$ QW degraded by more than a factor of 10 when moving the substrates from native GaAs to $\mathrm{Si}$ [73]. Two nominally identical laser structures (except for the active region) were grown as shown in the inset of Figures 6(a) and 6(b) and then fabricated into ridge-waveguide lasers using the same processing procedure. Despite similar current-voltage (I-V) characteristics obtained from those two different 
kinds of lasers [73], the behavior of RT continuous-wave (CW) light-current (L-I) characteristics between Si-based QDs and QW lasers was totally different. In accordance with the PL observations, CW lasing was achieved only from Si-based QD lasers, while none of the QW lasers grown Si were able to achieve RT CW lasing.

Innovations in QDs have spawned companies (for example, Innolume from Germany and QD Laser from Japan), and launched mass-production product lines for Data- and Telecommunications applications. Despite significant progress, however, QW lasers are still the main force in Data/Telecom, with QD lasers, at the moment, in a niche of the market. While the interest in QD lasers is growing and, in particular, III-V-on-Si integration presents incredible new opportunities for QD active regions, there is still some work to do to match the optimistic expectations.

\section{4. $1.3 \mu \mathrm{m} \operatorname{InAs} / \mathrm{GaAs} \mathrm{QD}$ edge-emitting lasers grown on $\mathrm{Si}$}

\subsection{Ge and Ge-on-Si substrates}

The epitaxial growth of GaAs on $\mathrm{Si}$ encounters significant hurdles because of the challenges discussed above in Section 2. To avoid these hurdles, many research efforts have been devoted to the use of Ge substrates. This development was triggered by a number of important properties exhibited by Ge substrates. First, the small lattice mismatch $(0.08 \%)$ and closely matched coefficient of thermal expansion between $\mathrm{Ge}$ and $\mathrm{Si}$ bridge the material difference gap between GaAs and Si substrate. Second, the higher mobilities for electrons and holes in Ge than Si and the large absorption coefficient at telecommunications wavelengths make it a good candidate for both electronic and photo-detection devices. Furthermore, Ge is compatible with Si COMS circuits and the fabrication of high-quality Geon-Si virtual substrates, has become a mature technology nowadays.

Recent advanced epitaxial techniques with monolithic integration of GaAs on Ge substrates and InAs/GaAs QDs gain region have enabled the realization of PL emission of InAs/GaAs QDs on a Ge substrate. However, the emission wavelength was limited at $\sim 1.1 \mu \mathrm{m}$ below RT, and no laser was achieved $[74,75]$. One of the biggest challenges in such an effort was the formation of APBs when the typical procedure of using a self-terminating arsenide (As) layer was adopted for the initial GaAs layer grown on a Ge substrate, see Figure 7(a). A breakthrough was made by Liu et al from UCL in 2011 by overcoming the APB issue through introducing a Ga prelayer technique for the initial GaAs growth, thus allowing the formation of a single-domain GaAs buffer layer, as shown in Figure 7(b) before subsequent epitaxy of III-V materials [76]. The improved material quality with the use of selfterminating Ga technique was also confirmed by TEM measurements as shown in Figures 7(c) and 7(d). Based on this template, an InAs/GaAs QD laser emitting at $1310 \mathrm{~nm}$ was directly grown on a $\mathrm{Ge}$ (100) substrate, with $6^{\circ}$ off-cut towards the [111] plane. This laser had a low threshold current density of $55.2 \mathrm{~A} / \mathrm{cm}^{2}$ as seen from Figure 7(e); comparable to the best previously reported values for QD lasers on native $\mathrm{GaAs}$, together with a maximum $\mathrm{CW}$ operation temperature of $60^{\circ} \mathrm{C}$ (see Figure 7(f)) [76].

Following the first demonstration of an electrically pumped $1.3 \mu \mathrm{m}$ InAs/GaAs QD laser on a $\mathrm{Ge}$ substrate, the first successful demonstration of an electrically-pumped CW $1.3 \mu \mathrm{m} \mathrm{InAs/GaAs} \mathrm{QD}$ laser grown directly on a Ge-on-Si virtual substrate followed soon after by the same research group [77]. In this work, as shown in Figure 8(a), the InAs/GaAs QD laser structure was directly grown on a Ge-on-Si virtual substrate by solid-source molecular beam epitaxy (MBE). The Ge-on-Si virtual 
substrate with a typical threading dislocation density of $\sim 5 \times 10^{6} \mathrm{~cm}^{-2}$ was fabricated by epitaxially depositing a $2-\mu \mathrm{m} p+$ Ge layer on phosphorus doped (100)-oriented Si substrates with a $6^{\circ}$ offcut towards the [111] plane using chemical vapour deposition illustrated in Figure 8 (b) (bottom). To form an high-quality GaAs buffer layer on a Ge-on-Si virtual substrate, the Ga prelayer technique discussed above [76] was again adopted to suppress the formation of APBs. This was followed by 20 monolayers of GaAs grown by migration enhanced epitaxy (MEE) and 2- $\mu \mathrm{m} p+\mathrm{GaAs}$ buffer layer as shown in Figure 8(b) (upper). As seen in Figure 8(c), RT CW lasing at a wavelength of $\sim 1280 \mathrm{~nm}$ was achieved from this device with a CW (pulsed) threshold current density of 163 (63.4) $\mathrm{A} / \mathrm{cm}^{2}$ and maximum CW (pulsed) lasing temperature up to $30(84){ }^{\circ} \mathrm{C}$, respectively. This method was later improved by A. Liu et al from UCSB, who demonstrated high-temperature CW lasing operation up to $119^{\circ} \mathrm{C}$ by employing p-type modulation doping to the QD active region, as shown in Figure 8(d) [78]. An initial reliability study of InAs/GaAs QD lasers grown directly on Ge-on-Si substrates was also performed [79]. The experimental results reveal that some devices maintained lasing oscillation over $2700 \mathrm{~h}$ of constant $\mathrm{CW}$ current stress at $30^{\circ} \mathrm{C}$, and that p-doped laser show the best lifetime, with an extrapolated mean time to failure (MTTF) of 4,627 h obtained from the presented aging data in Figure 8(e). This lifetime result was among the highest reported for any kind of III-V lasers on Si at that time. Moreover, no catastrophic failures were observed.

\subsection{Offcut Si substrates}

Despite the fact that using the Ge-on-Si virtual substrate demonstrated excellent laser performance, for direct integration with Si, achiveing a laser without the intermediate Ge buffer layer would be regarded as more attractive approach, since the existence of $\mathrm{Ge}$ on $\mathrm{Si}$ not only limits the range of $\mathrm{Si}$ circuits but also restricts the light coupling into a $\mathrm{Si}$ waveguide through this layer because of the large optical absorption coefficient of $\mathrm{Ge}$ at telecommunications wavelengths [27]. Consequently, returning to $\mathrm{Si}$ substrates without a Ge buffer layer remains the ultimate goal enabling reinvigoration of $\mathrm{Si}$ photonics.

The first QD laser monolithically grown on Si was reported by Linder et al in 1999 [80]. In this work, self-organized $\operatorname{In}_{0.4} \mathrm{Ga}_{0.6}$ As QDs were used as the active region to achieve lasing at $\sim 1 \mu \mathrm{m}$. However, due to the existence of a high density of dislocations, this device could only operate under pulsed operation at a low temperature of $80 \mathrm{~K}$, and with a very high threshold current density of $3.85 \mathrm{kA} / \mathrm{cm}^{2}$. A few years later, in 2005 , the demonstration of $\operatorname{In}_{0.5} \mathrm{Ga}_{0.5} \mathrm{As}$ QD lasers operating at $1 \mu \mathrm{m}$ grown directly on Si substrates at RT was reported for the first time. The devices were characterized by threshold current density of $1.5 \mathrm{kA} / \mathrm{cm}^{2}$, output power of over $50 \mathrm{~mW}$, and large $\mathrm{T}_{0}$ of $244 \mathrm{~K}$ in the temperature range between 25 and $95^{\circ} \mathrm{C}$ [81]. However, it is worthwhile noting that the QD laser monolithically grown on Si for the long wavelengths $(1.3 \mu \mathrm{m}$ and $1.55 \mu \mathrm{m})$ was not realized until 2011 when Wang et al from UCL demonstrated the first $1.3 \mu \mathrm{m} \mathrm{InAs/GaAs} \mathrm{QD} \mathrm{laser} \mathrm{on} \mathrm{Si} \mathrm{[82].} \mathrm{In}$ this work, $1.3 \mu \mathrm{m} \mathrm{InAs/GaAs} \mathrm{QD} \mathrm{lasers} \mathrm{were} \mathrm{directly} \mathrm{integrated} \mathrm{on} \mathrm{Si}(001)$ substrate with $4^{\circ}$ off-cut towards [011] plane. To minimize the high density of TDs, the growth of a GaAs nucleation layer (NL) was explored, which has proven to be effective in confining defects at the interface between the GaAs buffer and Si substrate. As a result, this device achieved RT lasing under pulsed operation at $1300 \mathrm{~nm}$ with a threshold current density of $725 \mathrm{~A} / \mathrm{cm}^{2}$ and a maximum lasing temperature of $42^{\circ} \mathrm{C}$. Since this pioneering work, numerous approaches have been investigated, by the same research group to optimise the III-V buffers with gradually reduced TDs density, including AlAs NL [83], combination of InAlAs/GaAs dislocation filter layers (DFLs) and GaAs NL [84, 85], and thermal annealing of DFLs [86]. As a result, InAs/GaAs QD lasers epitaxially grown directly on Si substrates have been demonstrated with substantial improvement of their device characteristics. Although QD lasers on Si 
substrates have shown superior performance in the last few years, earlier research results [87, 88] indicated that the quality of GaAs on Si substrates was much inferior with that on native GaAs substrates, in terms of defect density, typically over $2 \times 10^{6} \mathrm{~cm}^{-2}$ for a Si substrate compared with $\sim 1$ $\times 10^{3}$ to $1 \times 10^{4} \mathrm{~cm}^{-2}$ for a GaAs substrate [89].

A considerable advance towards a practical QD laser directly grown on a Si substrate has been made by Chen et al from UCL in 2016 who demonstrated high-performance $1.3 \mu \mathrm{m}$ InAs/GaAs QD lasers on a $\mathrm{Si}$ substrate with long extrapolated lifetime [27]. In this work, InAs/GaAs QD lasers were directly grown on $\mathrm{Si}$ (100) substrates with $4^{\circ}$ offcut to the [011] plane in order to prevent the formation of APBs during the growth [90], as shown in Figure 9(a). To reduce the density of TDs, various growth techniques were adopted. For the initial growth stage, a $6 \mathrm{~nm}$-thick AlAs NL was employed, which is effective in suppressing three-dimensional growth and hence provides a superior interface quality between $\mathrm{Si}$ substrate and GaAs buffer layer [83], as shown in Figure 9(b). Subsequently, multi-step growth method was introduced to grow 1 um-thick GaAs buffer layers comprised of 30, 170 and $800 \mathrm{~nm}$-thick GaAs grown at 350, 450 and $590{ }^{\circ} \mathrm{C}$, respectively [39]. As a result, it was revealed that the AlAs NL and multi-step growth effectively confined the most of defects at the first $200 \mathrm{~nm}$ of GaAs buffer layer (position 1 in Figure 9(c)). Nonetheless, the high density of TDs propagating toward active region still remained on the order of $10^{9} \mathrm{~cm}^{-2}$. To further reduce the density of TDs, strained-layer superlattices (SLSs) consisting of $\operatorname{In}_{0.18} \mathrm{Ga}_{0.82} \mathrm{As} / \mathrm{GaAs}$ were employed as DFLs, providing a strong in-plane force and therefore promoting the motion of TDs with the misfit strain. A more detailed description for designing DFLs can be found in [87]. Furthermore, in situ thermal annealing cycle was implemented after the growth of each SLS, which enhances the mobility of TDs and thereby enables the TDs to meet and annihilate each other [86]. Figure 9(d) exhibits that after 4-layers of DFL, the density of TDs is significantly reduced from $10^{9} \mathrm{~cm}^{-2}$ to $10^{5}$ $\mathrm{cm}^{-2}$.

For the characterization of QD properties, several measurements were carried out. As shown in Figure 9(e), an atomic force microscopy (AFM) image exhibits that a high QD density of $\sim 3.0 \times 10^{10} \mathrm{~cm}^{-2}$ with an excellent uniformity was achieved in this work. A small inhomogeneous broadening of QDs was also evidenced by a narrow full-width at half-maximum (FWHM) of $\sim 29 \mathrm{meV}$ from RT PL in Figure 9(e). In addition, a high-angle annular dark-field scanning TEM image of single QD with diameter and height in $\sim 20$ and $\sim 7 \mathrm{~nm}$, respectively, indicates a uniform indium distribution with marginal intermixing in Figure 9(f). Consequently, owing to various strategies mentioned above for the high-quality III-V layers on $\mathrm{Si}$, most of defects were effectively suppressed and high quality of QDs in dot-in-well (DWELL) structure was obtained. More detailed description can be found in [27].

Due to the achievement of high-quality III-V epilayers as well as using QDs act as the active region, high-performance electrically pumped CW $1310 \mathrm{~nm}$ InAs/GaAs QD lasers directly grown on Si have been achieved, with a record low threshold current density of $62.5 \mathrm{~A} / \mathrm{cm}^{2}$ at RT, high power of over $100 \mathrm{~mW}$ and high-temperature operation up to $75^{\circ} \mathrm{C}\left(120^{\circ} \mathrm{C}\right.$ under pulsed operation) (See Figures 10 (a) and 10(b)) [27]. The uniformity of QDs is a critical parameter for the massive production and streamlined fabrication of QD-based lasers on Si. A histogram of measured threshold current densities for $70 \mathrm{Si}$-based QD lasers of the same dimension from different areas within a quarter 3-inch wafer was presented. As shown in Figure 10(c), the distribution corresponding to threshold current density of $67.5+/-17.5 \mathrm{~A} \mathrm{~cm}^{-2}$ was measured to be $90 \%$, implying highly uniform QD distribution. For practical applications, long-lifetime is inevitable. Over 3,100 h of $\mathrm{CW}$ operation was reported from the InAs QD laser on Si described above un der ACC mode at $26^{\circ} \mathrm{C}$ as seen from Figure 10 (d). By 
defining the lifetime, the time when the threshold becomes double, an extrapolated lifetime of over $100,158 \mathrm{~h}$ was estimated by fitting the threshold with a sublinear model [79].

\subsection{On-axis $\mathrm{Si}(001)$ substrates}

For previous pioneering works, the high-performance QD lasers were grown on Si substrates with an offcut of $4^{\circ}-6^{\circ}$ to the [110] plane $[27,77-79,82]$, which could form double-atomic-height steps on $\mathrm{Si}$ surface and effectively avoid APBs formation during the growth of polar III-V on non-polar Si [36]. Even though this approach successfully inhibits the emergence of the APBs, to fully exploit the deployment of Si photonics through conventional CMOS-compatible foundries, it is imperative to use nominal (001) Si substrates with a miscut angle less than $0.5^{\circ}$, namely, the so-called "exact" (001) Si substrates $[91,92]$.

The first successful approach for realizing an electrically pumped $1.3 \mu \mathrm{m} \mathrm{CW} \mathrm{InAs/GaAs} \mathrm{QD} \mathrm{laser} \mathrm{on}$ on-axis Si (001) substrates was reported by Liu et al from UCSB in early 2017 [93]. In this study, a commercial $300 \mathrm{~mm} \mathrm{GaP} / \mathrm{Si}$ template, grown in an MOCVD by NAsP III-V GmbH, was used. The estimated miscut of the template was around $0.1-0.2^{\circ}$. To terminate APBs within the $45 \mathrm{~nm}$ GaP layer, the $\mathrm{Si}$ wafer was firstly subjected to a special pre-epitaxial heat treatment followed by a $200 \mathrm{~nm} \mathrm{Si}$ homo-epitaxy [92]. The wafer was then cut into small pieces and transferred into MBE chamber, where a $2.3 \mu \mathrm{m}$ GaAs buffer layer [94] was first grown directly on the GaP/Si template, followed by a thermal cycle annealing to facilitate the annihilation of TDs. Above the GaAs buffer, InAs/GaAs QDs active region [95] embedded in a GaAs/AlGaAs graded-index separate confinement heterostructure was then grown. The threading dislocation density (TDD) determined by electron channelling contrast imaging (ECCI) measurements was $\sim 3 \times 10^{8} \mathrm{~cm}^{-2}$. Both broad-area lasers and ridge-waveguide lasers were fabricated. A typical scanning electron microscope (SEM) image of a ridge-waveguide laser is shown in Figure 11(a). Broad-area lasers with uncoated facets achieved RT CW lasing with threshold current densities of $\sim 860 \mathrm{~A} / \mathrm{cm}^{2}$ and single-facet output power of over $100 \mathrm{~mW}$. Ridge-waveguide lasers with high-reflection (HR) coated facets operated CW with low threshold current down to 30 $\mathrm{mA}$ at RT and achieved maximum $\mathrm{CW}$ lasing temperatures up to $90^{\circ} \mathrm{C}$ [93]. This method was later improved by the same research group using optimized III-V buffers between the device layers and GaP/Si template [96], including in-situ thermal cycle annealing [96] and strained superlattices [87, 97]. As a result, the TDD has been reduced to $\sim 7 \times 10^{6} \mathrm{~cm}^{-2}$, a factor of 40 lower than previous unoptimized III-V buffer layers [96]. The ridge-waveguide lasers with as-cleaved facets fabricated from the optimized III-V buffers achieved CW threshold currents as low as $9.5 \mathrm{~mA}$ at $20{ }^{\circ} \mathrm{C}$ and $\mathrm{CW}$ operation up to $80^{\circ} \mathrm{C}$. Applying HR coating to one facet further reduced the $\mathrm{CW}$ threshold currents to $6.2 \mathrm{~mA}$ and improved lasing operation temperature to $85^{\circ} \mathrm{C}$ as shown in Figures 11 (b) and 11(c) [96]. The GaP/Si-based InAs QD lasers also showed a high CW wall-plug-efficiency (WPE) and high injection efficiency of $38.4 \%$ and $87 \%$ (see Figure 11(b)), respectively. Moreover, this approach was also used to successfully demonstrate excellent device lifetimes (see Figure 11(d)) with extrapolated MTTF of more than a million hours [98], which is a record-long value for GaAs-based laser grown on Si. A more detailed, updated summary of some representative lifetime data for GaAs-based lasers grown on Si that have been reported so far is shown in Table I [79].

Another direct growth approach uses GaAs-on-v-groove Si (GoVS) template [99] instead of a GaP intermediate buffer layer to prevent the formation of APBs and to reduce the TDD of epitaxial GaAs layer. In this case, the $\mathrm{N}$-doped on-axis $\mathrm{Si}$ substrates were firstly patterned with $\mathrm{SiO}_{2}$ stripes using standard dry etching techniques, followed by an RCA-1 clean (sometime called 'standard clean-1' developed by Werner Kern at RCA laboratories) and a rapid HF (1\%) dip to remove the native oxide 
before the immediately wet etching the v-grooves using KOH solutions [100]. The epitaxial growth of GaAs on the patterned v-groove substrate was performed by MOCVD in the following sequence: thermal cleaning of the patterned Si substrates, selective-area heteroepitaxy of GaAs nanowires [101], $\mathrm{SiO}_{2}$ stripe removal, coalescence of $\mathrm{GaAs}$ to form GaAs planar film, growth of superlattices, and finally growth of GaAs buffer layer to complete the growth of GoVS template. The great promise of this growth method comes not only from the ability to form a unique defect trapping effect [see the inset of Figure 12(a)] [36, 102] but also from the removal of the difficulties in achieving coalescence over dielectric patterns [36]. AFM, XRD, and ECCI measurements have been employed to assess the quality of GoVS template and have revealed a root-mean-square (RMS) roughness of $0.9 \mathrm{~nm}$, a FWHM of 140 arcsec and a TDD of $\sim 10^{7} \mathrm{~cm}^{-2}$, respectively [36], indicating a good template quality. Above this GoVS template, III-V buffer layer with optimized DFLs was grown to further improve the material quality; this was then followed by a standard InAs/GaAs QD laser structure. Following demonstration of RT PL emission at $1.3 \mu \mathrm{m}$ from InAs/GaAs QD structures grown directly on GoVS templates [103], the successful demonstration of an electrically pumped CW InAs/GaAs QD laser grown on a GoVS template was achieved [99], with a lasing threshold current as low as $37 \mathrm{~mA}$ and $\mathrm{CW}$ operation up to $80^{\circ} \mathrm{C}$ as seen from Figures $12(\mathrm{~b})$ and $12(\mathrm{c})$.

High-quality epitaxial electrically pumped $1.3 \mu \mathrm{m} \mathrm{CW}$ InAs/GaAs QD lasers have also been achieved by Chen et al from UCL through direct GaAs growth without the use of any intermediate buffer layers or patterned Si substrates [104]. In this work, APB-free GaAs films were directly grown on $300 \mathrm{~nm}$ COMS-compatible Si (001) substrates by MOCVD [38]. One of the biggest challenges in such an effort is developing methodologies that allow the formation of double-atomic-height steps for quasinominal Si (001), otherwise, as seen from Figure 13(a), high density of APBs can be observed, inducing a high surface roughness of $1.6 \mathrm{~nm}$ [38]. To solve these problems, in the initial growth step, on-axis $\mathrm{Si}(001)$ wafers were deoxidized by the SiConi process using a $\mathrm{NF}_{3} / \mathrm{NH}_{3}$ remote plasma, and then transferred into MOCVD reactor to perform $\mathrm{H}_{2}$ annealing at $900{ }^{\circ} \mathrm{C}$, forming $2 \times 1$ surface structure (see Figure 13(b)). Subsequently, the chamber was then quickly cooled down to $700{ }^{\circ} \mathrm{C}$ within 30s to freeze the Si surface structure. Finally, a standard two-step process was carried out to grow GaAs layers. As seen from Figure 13(c), this approach has enabled the formation of APBs-free GaAs layer with a surface roughness of $0.8 \mathrm{~nm}$ [38]. Note that this roughness is comparable to the lowest value achieved for $1 \mu \mathrm{m}$ thick GaAs layer grown on off-cut Si substrates [105-107], whereas only $150 \mathrm{~nm}$ GaAs was grown on the on-axis Si (001) substrates. By exploiting this template and previously optimized III-V buffer layers, InAs/GaAs QD structures grown directly on this virtual $\mathrm{GaAs} / \mathrm{Si}$ (001) have shown comparable PL intensity as compared to those grown on native GaAs substrate [104]. Consequently, electrically-pumped lasers fabricated on this on-axis Si (001) substrates exhibited threshold current densities of $\sim 425 \mathrm{~A} / \mathrm{cm}^{2}$ with output power over $40 \mathrm{~mW}$ at RT [see Figure 13(d)]. Under pulsed operation, lasing operation up to $102{ }^{\circ} \mathrm{C}$ (see Figure 13(e)) has been realized, with a threshold current density of $250 \mathrm{~A} / \mathrm{cm}^{2}$ and single facet output power exceeding 130 $\mathrm{mW}$ at RT. More recently, $1250 \mathrm{~nm}$ InAs/GaAs QD lasers monolithically grown on on-axis $\mathrm{Si}$ (100) was demonstrated by using only MBE process [108]. In this work, a $40 \mathrm{~nm} \mathrm{Al}_{0.3} \mathrm{Ga}_{0.7} \mathrm{As}$ seed layer was grown on the $\mathrm{Si}(001)$ substrate at relative high temperature $\left(500{ }^{\circ} \mathrm{C}\right)$ and high growth rate of 1.1 $\mu \mathrm{m} / \mathrm{h}$. The APBs could be annihilated within the following GaAs buffer layer of less than $400 \mathrm{~nm}$. This approach offers potential benefits of simple growth process for fabricating QD lasers monolithically on CMOS-compatible Si (001) substrates. A more detailed, updated summary of some representative milestone of III-V/IV QD edge-emitting lasers by monolithic integration is presented in Table II [109]. 


\section{O-band distributed-feedback (DFB) laser array monolithically grown on $\mathrm{Si}$}

While the realization of high-performance Si-based electrically-pumped Fabry-Perot (FP) laser sources is an enabling technology for single channel optical interconnects, advanced communications systems for wavelength division multiplexing (WDM) necessitate arrays of multiple single-mode lasers [110]. Recently, with the development of a selective-area growth technique, it was reported that high-quality InP-based material was directly grown on buffer-free Si substrate [111]. Based on this technique, researchers from Ghent University and IMEC have demonstrated the first InP-based DFB laser array monolithically grown on $\mathrm{Si}$ [112]. However, the operating wavelength of this array was around $920 \mathrm{~nm}$, which is not compatible with $\mathrm{Si}$ waveguides. This problem was, soon after, tackled by the same research group, using an $\mathrm{InP} / \mathrm{InGaAs} / \mathrm{InP}$ heterostructure as the active region grown directly on the aforementioned buffer-free InP-on-Si virtual substrates. Consequently, they have realized the first O-band DFB laser array monolithically grown on $\mathrm{Si}$ [113]. Despite these significant achievements, these DFB arrays have several key challenges need to be solved, such as operation under only optical pumping and the limited maximum wavelength coverage of $40 \mathrm{~nm}$ [113].

Very recently, a breakthrough has been made in O-band DFB laser array by researchers at the UCL in the UK and the Sun Yat-sen University in China, who not only reported the first electrically-pumped DFB laser array on a Si substrate, but also presented a record wavelength coverage of $100 \mathrm{~nm}$ [114]. In this work, an InAs/GaAs QD laser structure was directly grown on an n-doped $\mathrm{Si}(001)$ substrate with a $4^{\circ}$ off-cut angle towards the [011] plane following previously optimized growth conditions. To avoid complicated regrowth that has normally been adopted for development of conventional III-V DFB lasers, here, lateral surface gratings were employed and fabricated at the same time as the waveguide etching process was conducted by using an inductively coupled plasma (ICP) dry etching, as shown in Figure 14(a). The first-order sidewall gratins with a $\lambda / 4$ phase shift (see Figure 14(a)) were designed in order to provide single-longitudinal-mode lasing. In addition, the suppression of facet back reflections is also required as another important parameter for DFB lasers, otherwise leading to mode jumping at high currents. To overcome this problem cost-effectively, instead of using anti-reflection (AR) coated laser facets, AR output couplers were designed and fabricated by carefully optimizing the tilting angle and waveguide width. As a result, the reflection coefficient was successfully decreased down to $\sim 1 \%$ and the output efficiency was improved up to $\sim 82 \%$, meanwhile maintaining a reasonable beam profile that can be coupled into an optical fiber.

By combining III-V QD gain region technology, advanced epitaxy and high-end nano-fabrication techniques discussed above, the first electrically-pumped RT CW single-mode DFB laser array has been fabricated. Figure 14(b) shows partially the schematics of the individual DFB laser. As seen in Figures 14(c) and 14(d), individual devices in the array produced low CW threshold currents of 12 $\mathrm{mA}$ and high single-mode side mode suppression ratios (SMSRs) of $50 \mathrm{~dB}$ [114]. The laser array operated at O-band covering a record wavelength range of $100 \mathrm{~nm}$ (see Figure 14(d)). The key behind such a broad wavelength coverage was attributed to the ultra-broad gain bandwidth enabled by the inhomogeneity of QDs. Moreover, a $20 \pm 0.2 \mathrm{~nm}$ channel spacing was achieved, which can be applied to the standard coarse wavelength division multiplex (CWDM) grid [114]. These results provide potential building blocks toward not only monolithically integrated Si-based PICs for optical communications, but also non-communications such as on-chip sensing and metrology in which single-mode coherent light sources play an important role. 


\section{InAs QD micro-lasers monolithically grown on $\mathrm{Si}$}

In addition to ridge-waveguide lasers, micro-lasers with small-size, low power consumption and a short cavity length have also shown great potential to serve as long wavelength light sources for $\mathrm{Si}$ photonic integration [115]. Micro-ring and micro-disk lasers using whispering gallery mode (WGM) resonance also ideally offer the higher stability of emission wavelength to the temperature variation in comparison with macro-laser [116]. There are several publications of optically pumped 1.3 and 1.5 $\mu \mathrm{m}$ InAs QD micro-disk lasers monolithically grown on Si [117-121]. For example, Wan et al demonstrated optically pumped $1.3 \mu \mathrm{m}$ RT InAs QD micro-disk lasers directly grown on (001) Si using V-grooved Si template and observed that the fabricated micro-disk laser produced RT CW lasing with a very low threshold power of $200 \mu \mathrm{W}$ for a five-layer QD [117]. To obtain APB-free GoVS template, GaAs thin films were formed by the coalescence of a well-ordered array of GaAs nanowires on Si substrates, leading to the formation of the high-quality GoVS template. In 2017, Shi et al reported CW optically pumped $1.5 \mu \mathrm{m}$ InAs/InAlGaAs microdisk QD laser grown on (001) $\mathrm{Si}$ substrates by employing InP buffer layer on $\mathrm{GaAs} / \mathrm{Si}$ template [120]. The threshold power of the fabricated laser was measured to be $1.6 \mathrm{~mW}$ at $4.5 \mathrm{~K}$. The observed lasing behavior can be attributed to InP buffer layer with 7-layer of QD dislocation filter layer.

For practical integration of Si photonics, however, electrically pumped lasers grown on Si substrates are imperative. Until now, there are few publications of electrically injected micro-lasers on $\mathrm{Si}$. The first demonstration of CW electrically pumped $1.3 \mu \mathrm{m}$ InAs/GaAs QD micro-disk laser grown on Si (001) substrates with $4^{\circ}$ offcut to the [110] plane was reported in 2017 by Kryzhanovskaya et al [122]. Figure 15(a) illustrates the schematic diagram of the layer structure of this micro-disk laser on Si. The III-V QD laser structure consisted of AlAs nucleation layer, GaAs buffer layer, AlGaAs/GaAs SLS as DFLs, seven InAs/GaAs DWELL structures sandwiched by two AlGaAs n- and p-type cladding layers, and highly doped p-type GaAs contacting layer. Under RT CW electrically pumping, lasing operation at $1.3 \mu \mathrm{m}$, see Figure 15(b) with a minimum threshold of $600 \mathrm{~A} / \mathrm{cm}^{2}$, a narrow linewidth of $30 \mathrm{pm}$ and low sensitivity to the current-introduced self-heating was achieved in this work. In addition, $\mathrm{CW}$ electrically pumped micro-ring lasers directly grown on nominal (001) Si substrates using Vgrooved, and $\mathrm{GaP} / \mathrm{Si}$ templates were also achieved [123, 124]. For instance, Wan et al investigated the effect of growth templates such as native GaAs, (001) GaP/Si, and V-groove (001) Si substrates on the overall performance of electrically pumped InAs QD micro-ring lasers [124]. This comprehensive comparison indicated that while the $\mathrm{GaP} / \mathrm{Si}$ template provides lower defect densities, the $\mathrm{V}$-grooved $\mathrm{Si}$ is beneficial in obtaining a smooth surface and thin buffer layers.

\section{Conclusion}

This review has predominantly discussed the recent progress made in the epitaxial growth of various types of III-V quantum dot (QD) lasers on Si substrates, which operate mainly at O-band for Si photonic optical interconnects, along with the strategies for overcoming the roadblocks induced by the material dissimilarity between III-Vs and Si substrates. QD gain technology is poised to revolutionize monolithic integration of III-V lasers on Si substrates, and a great deal of effort has been made to realize Si-based on-chip light-emitting sources comprising III-V InAs/GaAs QD structures. For QD lasers on $\mathrm{Ge}, \mathrm{Ge} / \mathrm{Si}$, and offcut $\mathrm{Si}$ substrates, to reduce the defect density, various approaches, including the development of nucleation layers, the optimization of dislocation filter layers, in situ thermal annealing process, have been fully investigated. Owing to such a huge effort, $1.3 \mu \mathrm{m}$ InAs QD lasers monolithically grown on offcut Si substrates achieved remarkable results, particularly, the low threshold current density of $62.5 \mathrm{~A} / \mathrm{cm}^{2}$, high operation temperature up to $120{ }^{\circ} \mathrm{C}$ and long 
extrapolated lifetime of over 100,000 hours. To take advantage of mature fabrication tools and processes developed by the Si CMOS industry, soon after, by leveraging techniques developed from off-cut $\mathrm{Si}$ substrates, there has been substantial achievements for lasing operation from on-axis $\mathrm{Si}$ (001) substrates through $\mathrm{GaP} / \mathrm{Si}$, V-grooved $\mathrm{Si}$, and $\mathrm{GaAs} / \mathrm{Si}$ templates. Now, $1.3 \mu \mathrm{m}$ InAs QD lasers monolithically grown on on-axis Si substrates have demonstrated threshold current as low as $6.2 \mathrm{~mA}$, high power of $185 \mathrm{~mW}$, wall-plug-efficiency and injection efficiency as high as $31 \%$ and $87 \%$, respectively, and extremely long extrapolated lifetime over a million hours for continuous-wave operation. Despite significant achievements by demonstrating high-performance ridge waveguide Fabry-Perot (FP) lasers epitaxially grown on both off-cut and on-axis Si (001) substrates, this achievement alone does not demonstrate the feasibility of using this new materials platform for creating lasers suitable for advanced communications systems, such as the QD distributed-feedback (DFB) laser array presented in this review paper, in which not only high-quality material but also advanced design and novel fabrication techniques are required. Consequently, by combining III-V InAs/GaAs QD gain region technology, advanced epitaxy, and advanced nano-fabrication techniques, the first monolithically integrated electrically-pumped DFB laser array on Si has been realized with a record wavelength coverage of $100 \mathrm{~nm}$ covering the whole O-band. This represents a key step in laser manufacturing and higher integration levels in Si photonics. In addition to ridge waveguide lasers, QD micro-lasers grown Si substrates have also been extensively studied. Electrically pumped QD microlasers on $\mathrm{Si}$ were demonstrated very recently, which, can be, to a large extent, attributed to the advanced growth strategies developed in previous FP QD lasers on Si.

So far, the achievements made in monolithic Si-based QD lasers represents a major step towards the realization of cost-effective high-volume optical interconnects. The next step is the integration of onchip lasers with other Si-based photonic components, both actively and passively, to develop end-toend $\mathrm{Si}$ photonics integrated links by the monolithic method. This is a very exciting and, of course, more challenging task, which requires huge efforts from Si photonics community.

Acknowledgment: The authors acknowledge financial support from UK EPSRC under Grant No. EP/P000886/1, Grant No EP/P006973/1, and EPSRC National Epitaxy Facility. M.L. thanks the Chinese Scholarship Council for funding her research studies. S.C. thanks the Royal Academy of Engineering for funding his Research Fellowship.

\section{Reference:}

[1] Rickman A 2014 The commercialization of silicon photonics Nat. Photonics 8 579-82

[2] Reed G T, Mashanovich G, Gardes F Y and Thomson D J 2010 Silicon optical modulators Nat. Photonics 4 518-26

[3] Xiao X, Xu H, Li X, Li Z, Chu T, Yu Y and Yu J 2013 High-speed, low-loss silicon MachZehnder modulators with doping optimization Opt. Express 214116

[4] Streshinsky M, Ding R, Liu Y, Novack A, Yang Y, Ma Y, Tu X, Chee E, Lim A, Lo P, Baehr-Jones T and Hochberg M 2013 Low power $50 \mathrm{~Gb} / \mathrm{s}$ silicon traveling wave MachZehnder modulator near $1300 \mathrm{~nm}$ Opt. Express 2130350

[5] Dosunmu O I, Cannon D D, Emsley M K, Kimerling L C and Ünlü M S 2005 High-speed resonant cavity enhanced Ge photodetectors on reflecting Si substrates for 1550-nm operation IEEE Photonics Technol. Lett. 17 175-7

[6] Yin T, Cohen R, Morse M M, Sarid G, Chetrit Y, Rubin D and Paniccia M J 200731 GHz Ge n-i-p waveguide photodetectors on Silicon-on-Insulator substrate Opt. Express 1513965

[7] Vivien L, Polzer A, Marris-Morini D, Osmond J, Hartmann J M, Crozat P, Cassan E, Kopp C, Zimmermann H and Fédéli J 2012 Zero-bias 40Gbit/s germanium waveguide photodetector on 
silicon Opt. Express 201096

[8] Bauters J, Davenport M, Heck M, Doylend J, Chen A, Fang A and Bowers J 2013 Silicon on ultra-low-loss waveguide photonic integration platform Opt. Express 21544

[9] Heck M, Bauters J, Davenport M, Spencer D and Bowers J 2014 Ultra-low loss waveguide platform and its integration with silicon photonics Laser Photon. Rev. 8 667-86

[10] Zheng X, Shubin I, Li G, Pinguet T, Mekis A, Yao J, Thacker H, Luo Y, Costa J, Raj K, Cunningham J E and Krishnamoorthy A V. 2010 A tunable 1x4 silicon CMOS photonic wavelength multiplexer/demultiplexer for dense optical interconnects Opt. Express 185151

[11] Tan D, Ikeda K, Zamek S, Mizrahi A, Nezhad M, Krishnamoorthy A, Raj K, Cunningham J, Zheng X, Shubin I, Luo Y and Fainman Y 2011 Wide bandwidth, low loss 1 by 4 wavelength division multiplexer on silicon for optical interconnects Opt. Express 192401

[12] Liang D and Bowers J E 2010 Recent progress in lasers on silicon Nat. Photonics 4 511-7

[13] Rong H, Liu A, Jones R, Cohen O, Hak D, Nicolaescu R, Fang A and Paniccia M 2005 An all-silicon Raman laser Nature 433 292-4

[14] Liu J, Sun X, Camacho-Aguilera R, Kimerling L and Michel J 2010 Ge-on-Si laser operating at room temperature Opt. Lett. 35679

[15] Wirths S, Geiger R, von den Driesch N, Mussler G, Stoica T, Mantl S, Ikonic Z, Luysberg M, Chiussi S, Hartmann J M, Sigg H, Faist J, Buca D and Grutzmacher D 2015 Lasing in directbandgap GeSn alloy grown on Si Nat. Photonics 9 88-92

[16] Park H, Fang A, Kodama S and Bowers J 2005 Hybrid silicon evanescent laser fabricated with a silicon waveguide and III-V offset quantum wells Opt. Express 139460

[17] Chang H-H, Fang A W, Sysak M N, Park H, Jones R, Cohen O, Raday O, Paniccia M J and Bowers J E 2007 1310Nm Silicon Evanescent Laser Opt. Express 1511466

[18] Fang A, Park H, Cohen O, Jones R, Paniccia M and Bowers J 2006 Electrically pumped hybrid AlGaInAs-silicon evanescent laser Opt. Express 149203

[19] Tanaka S, Jeong S, Sekiguchi S, Kurahashi T, Tanaka Y and Morito K 2012 High-outputpower, single-wavelength silicon hybrid laser using precise flip-chip bonding technology Opt. Express 2028057

[20] Tanabe K, Watanabe K and Arakawa Y 2012 III-V/Si hybrid photonic devices by direct fusion bonding. Sci. Rep. 2349

[21] Zhou Z, Yin B and Michel J 2015 On-chip light sources for silicon photonics Light Sci. Appl. 4 e358-e358

[22] Wang Z, Abbasi A, Dave U, De Groote A, Kumari S, Kunert B, Merckling C, Pantouvaki M, Shi Y, Tian B, Van Gasse K, Verbist J, Wang R, Xie W, Zhang J, Zhu Y, Bauwelinck J, Yin X, Hens Z, Van Campenhout J, Kuyken B, Baets R, Morthier G, Van Thourhout D and Roelkens G 2017 Novel Light Source Integration Approaches for Silicon Photonics Laser Photon. Rev. 111700063

[23] Liao M, Chen S, Huo S, Chen S, Wu J, Tang M, Kennedy K, Li W, Kumar S, Martin M, Baron T, Jin C, Ross I, Seeds A and Liu H 2017 Monolithically Integrated Electrically Pumped Continuous-Wave III-V Quantum Dot Light Sources on Silicon IEEE J. Sel. Top. Quantum Electron. 23 1-10

[24] Rafailov E U, Cataluna M A and Sibbett W 2007 Mode-locked quantum-dot lasers Nat. Photonics 1 395-401

[25] Nishi K, Takemasa K, Sugawara M and Arakawa Y 2017 Development of Quantum Dot Lasers for Data-Com and Silicon Photonics Applications IEEE J. Sel. Top. Quantum Electron. $231-7$

[26] Beanland R, Sánchez A M, Childs D, Groom K M, Liu H Y, Mowbray D J and Hopkinson M 2008 Structural analysis of life tested $1.3 \mu \mathrm{m}$ quantum dot lasers J. Appl. Phys. 103014913

[27] Chen S, Li W, Wu J, Jiang Q, Tang M, Shutts S, Elliott S N, Sobiesierski A, Seeds A J, Ross I, Smowton P M and Liu H 2016 Electrically pumped continuous-wave III-V quantum dot lasers on silicon Nat. Photonics $10307-11$

[28] Fischer R, Masselink W T, Klem J, Henderson T, Mcglinn T C, Klein M V, Morkoc H, Mazur J H and Washburn J 1985 Growth and Properties of GaAs/AlGaAs on Nonpolar Substrates Using Molecular-Beam Epitaxy J. Appl. Phys. 58 374-81

[29] Wang W I 1984 Molecular beam epitaxial growth and material properties of GaAs and 
AlGaAs on Si (100) Appl. Phys. Lett. 44 1149-51

[30] Sugo M, Mori H, Itoh Y, Sakai Y and Tachikawa M $19911.5 \mu \mathrm{m}$-Long-Wavelength Multiple Quantum Well Laser on a Si Substrate Jpn. J. Appl. Phys. 30 3876-8

[31] Yamada T, Tachikawa M, Sasaki T, Mori H and Kadota Y 19987000 h continuous wave operation of multiple quantum well laser on Si at $50^{\circ} \mathrm{C}$ Appl. Phys. Lett. 701614

[32] Tournié E, Cerutti L, Rodriguez J-B, Liu H, Wu J and Chen S 2016 Metamorphic III-V semiconductor lasers grown on silicon MRS Bull. $41218-23$

[33] Zimmermann H 2010 Integrated silicon optoelectronics (Berlin: Springer)

[34] Wu J, Chen S, Seeds A and Liu H 2015 Quantum dot optoelectronic devices: lasers, photodetectors and solar cells J. Phys. D. Appl. Phys. 48363001

[35] Hamers R J, Tromp R M and Demuth J E 1986 Scanning tunneling microscopy of Si(001) Phys. Rev. B 34 5343-57

[36] Li Q and Lau K M 2017 Epitaxial growth of highly mismatched III-V materials on (001) silicon for electronics and optoelectronics Prog. Cryst. Growth Charact. Mater. 63 105-20

[37] Brammertz G, Mols Y, Degroote S, Motsnyi V, Leys M, Borghs G and Caymax M 2006 Lowtemperature photoluminescence study of thin epitaxial GaAs films on Ge substrates J. Appl. Phys. 99093514

[38] Alcotte R, Martin M, Moeyaert J, Cipro R, David S, Bassani F, Ducroquet F, Bogumilowicz Y, Sanchez E, Ye Z, Bao X Y, Pin J B and Baron T 2016 Epitaxial growth of antiphase boundary free $\mathrm{GaAs}$ layer on $300 \mathrm{~mm} \mathrm{Si}(001)$ substrate by metalorganic chemical vapour deposition with high mobility APL Mater. 4

[39] Akiyama M, Kawarada Y and Kaminishi K 1984 Growth of Single Domain GaAs Layer on (100)-Oriented Si Substrate by MOCVD Jpn. J. Appl. Phys. 23 L843-5

[40] Fang S, Adomi K, Iyer S, Morkoç H, Zabel H, Choi C and Otsuka N 1990 Gallium arsenide and other compound semiconductors on silicon J. Appl. Phys. 68 R31-58

[41] Yang V, Groenert M, Leitz C, Pitera A, Currie M and Fitzgerald E 2003 Crack formation in GaAs heteroepitaxial films on Si and SiGe virtual substrates J. Appl. Phys. 93 3859-65

[42] H Kroemer 1963 A proposed class of hetero-junction injection lasers Proc. IEEE 51 1782-3

[43] ALFEROV Z 1970 AlAs-GaAs heterojunction injection lasers with a low room-temperature threshold Sov. Phys. Semicond. 3 1107-10

[44] Arakawa Y and Sakaki H 1982 Multidimensional Quantum Well Laser and TemperatureDependence of Its Threshold Current Appl. Phys. Lett. 40 939-41

[45] Grundmann M, Richter U, Ustinov V M, Kop'ev P S, Kirstaedter N, Bimberg D, Werner P, Ruvimov S S, Ledentsov N N, Gösele U, Alferov Z I, Heydenreich J and Maximov M V 1994 Low threshold, large To injection laser emission from (InGa)As quantum dots Electron. Lett. 30 1416-7

[46] Mirin R, Gossard A and Bowers J 1996 Room temperature lasing from InGaAs quantum dots Electron. Lett. 321732

[47] Park G, Shchekin O B, Csutak S, Huffaker D L and Deppe D G 1999 Room-temperature continuous-wave operation of a single-layered $1.3 \mu \mathrm{m}$ quantum dot laser Appl. Phys. Lett. 75 3267

[48] Huang X, Stintz A, Hains C P, Liu G T, Cheng J and Malloy K J 2000 Very low threshold current density room temperature continuous-wave lasing from a single-layer InAs quantumdot laser IEEE Photonics Technol. Lett. 12 227-9

[49] Park G, Shchekin O B, Huffaker D L and Deppe D G 2000 Low-threshold oxide-confined 1.3- $\mu \mathrm{m}$ quantum-dot laser IEEE Photonics Technol. Lett. 12 230-2

[50] Liu H, Hopkinson M, Harrison C, Steer M, Frith R, Sellers I, Mowbray D and Skolnick M 2003 Optimizing the growth of $1.3 \mu \mathrm{m}$ InAs/InGaAs dots-in-a-well structure J. Appl. Phys. 93 2931-6

[51] Liu H, Sellers I, Badcock T, Mowbray D, Skolnick M, Groom K, Gutiérrez M, Hopkinson M, $\mathrm{Ng} \mathrm{J}$, David J and Beanland R 2004 Improved performance of $1.3 \mu \mathrm{m}$ multilayer InAs quantum-dot lasers using a high-growth-temperature GaAs spacer layer Appl. Phys. Lett. 85 704-6

[52] Freisem S, Ozgur G, Shavritranuruk K, Chen H and Deppe D G 2008 Very-low-threshold current density continuous-wave quantum-dot laser diode Electron. Lett. 44679 
[53] Shchekin O B and Deppe D G $20021.3 \mu \mathrm{m}$ InAs quantum dot laser with To=161 K from 0 to $80{ }^{\circ} \mathrm{C}$ Appl. Phys. Lett. 80 3277-9

[54] Sandall I, Smowton P, Walker C, Badcock T, Mowbray D, Liu H and Hopkinson M 2006 The effect of p doping in InAs quantum dot lasers Appl. Phys. Lett. 88111113

[55] Sugawara M and Usami M 2009 Quantum dot devices: Handling the heat Nat. Photonics 3 $30-1$

[56] Nishi K, Saito H, Sugou S and Lee J S 1999 A narrow photoluminescence linewidth of 21 $\mathrm{meV}$ at $1.35 \mu \mathrm{m}$ from strain-reduced InAs quantum dots covered by In0.2Ga0.8As grown on GaAs substrates Appl. Phys. Lett. 74 1111-3

[57] Otsubo K, Hatori N, Ishida M, Okumura S, Akiyama T, Nakata Y, Ebe H, Sugawara M and Arakawa Y 2004 Temperature-Insensitive Eye-Opening under 10-Gb/s Modulation of 1.3- $\mu \mathrm{m}$ P-Doped Quantum-Dot Lasers without Current Adjustments Jpn. J. Appl. Phys. 43 L1124-6

[58] Takada K, Tanaka Y, Matsumoto T, Ekawa M, Song H Z, Nakata Y, Yamaguchi M, Nishi K, Yamamoto T, Sugawara M and Arakawa Y 2011 Wide-temperature-range $10.3 \mathrm{Gbit} / \mathrm{s}$ operations of $1.3 \mu \mathrm{m}$ high-density quantum-dot DFB lasers Electron. Lett. 47206

[59] Buckley S, Rivoire K and Vučković J 2012 Engineered quantum dot single-photon sources Reports Prog. Phys. 75126503

[60] Liu A Y, Komljenovic T, Davenport M L, Gossard A C and Bowers J E 2017 Reflection sensitivity of $13 \mu \mathrm{m}$ quantum dot lasers epitaxially grown on silicon Opt. Express 259535

[61] Huyet G, O'Brien D, Hegarty S P, McInerney J G, Uskov A V., Bimberg D, Ribbat C, Ustinov V M, Zhukov A E, Mikhrin S S, Kovsh A R, White J K, Hinzer K and SpringThorpe A J 2004 Quantum dot semiconductor lasers with optical feedback Phys. status solidi 201 34552

[62] O'Brien D, Hegarty S P, Huyet G and Uskov A V. 2004 Sensitivity of quantum-dot semiconductor lasers to optical feedback Opt. Lett. 291072

[63] Capua A, Rozenfeld L, Mikhelashvili V, Eisenstein G, Kuntz M, Laemmlin M and Bimberg D 2007 Direct correlation between a highly damped modulation response and ultra low relative intensity noise in an InAs/GaAs quantum dot laser Opt Express 15 5388-93

[64] Gubenko A, Krestnikov I, Livshtis D, Mikhrin S, Kovsh A, West L, Bornholdt C, Grote N and Zhukov A 2007 Error-free $10 \mathrm{Gbit} / \mathrm{s}$ transmission using individual Fabry-Perot modes of lownoise quantum-dot laser Electron. Lett. 431430

[65] Akrout A, Shen A, Brenot R, Van Dijk F, Legouezigou O, Pommereau F, Lelarge F, Ramdane A and Guang-Hua Duan 2009 Separate Error-Free Transmission of Eight Channels at $10 \mathrm{~Gb} / \mathrm{s}$ Using Comb Generation in a Quantum-Dash-Based Mode-Locked Laser IEEE Photonics Technol. Lett. 21 1746-8

[66] Zhou Y-G, Zhou C, Cao C-F, Du J-B, Gong Q and Wang C 2017 Relative intensity noise of InAs quantum dot lasers epitaxially grown on Ge Opt. Express 2528817

[67] Zarifkar A and Sanaee M 2015 Theoretical Modeling of Relative Intensity Noise in p-Doped 1.3- $\mu \mathrm{m}$ InAs/GaAs Quantum Dot Lasers J. Light. Technol. Vol. 33, Issue 1, pp. 234-243 33 234-43

[68] Pawlus R, Columbo L L, Bardella P, Breuer S and Gioannini M 2018 Intensity noise behavior of an InAs/InGaAs quantum dot laser emitting on ground states and excited states Opt. Lett. 43 867

[69] Mi Z T, Yang J, Bhattacharya P, Qin G X and Ma Z Q 2009 High-Performance Quantum Dot Lasers and Integrated Optoelectronics on Si Proc. Ieee 97 1239-49

[70] Ovid'ko I 2002 Relaxation Mechanisms in Strained Nanoislands Phys. Rev. Lett. 88046103

[71] Yang J, Bhattacharya P and Mi Z 2007 High-performance In0.5Ga0.5As/GaAs quantum-dot lasers on silicon with multiple-layer quantum-dot dislocation filters IEEE Trans. Electron Devices 54 2849-55

[72] Shi B, Li Q and Lau K M 2017 Self-organized InAs/InAlGaAs quantum dots as dislocation filters for InP films on (001) Si J. Cryst. Growth 464 28-32

[73] Liu A Y, Srinivasan S, Norman J, Gossard A C and Bowers J E 2015 Quantum dot lasers for silicon photonics [Invited] Photonics Res. 3

[74] Banerjee S, Halder N and Chakrabarti S 2010 Stranski-Krastanow growth of multilayer $\mathrm{In}(\mathrm{Ga}) \mathrm{As} / \mathrm{GaAs}$ QDs on Germanium substrate Appl. Phys. A 99 791-5 
[75] Dhawan T, Tyagi R, Bag R K, Singh M, Mohan P, Haldar T, Murlidharan R and Tandon R 2010 Growth of InAs Quantum Dots on Germanium Substrate Using Metal Organic Chemical Vapor Deposition Technique Nanoscale Res. Lett. 5 31-7

[76] Liu H, Wang T, Jiang Q, Hogg R, Tutu F, Pozzi F and Seeds A 2011 Long-wavelength InAs/GaAs quantum-dot laser diode monolithically grown on Ge substrate Nat. Photonics 5 416-9

[77] Lee A, Jiang Q, Tang M, Seeds A and Liu H 2012 Continuous-wave InAs/GaAs quantum-dot laser diodes monolithically grown on Si substrate with low threshold current densities Opt. Express 20 22181-7

[78] Liu A Y, Zhang C, Norman J, Snyder A, Lubyshev D, Fastenau J M, Liu A W K, Gossard A $\mathrm{C}$ and Bowers J E 2014 High performance continuous wave $1.3 \mu \mathrm{m}$ quantum dot lasers on silicon Appl. Phys. Lett. 104 3-7

[79] Liu A, Herrick R, Ueda O, Petroff P, Gossard A and Bowers J 2015 Reliability of InAs/GaAs Quantum Dot Lasers Epitaxially Grown on Silicon IEEE J. Sel. Top. Quantum Electron. 21

[80] Linder K K, Phillips J, Qasaimeh O, Liu X F, Krishna S, Bhattacharya P and Jiang J C 1999 Self-organized In0.4Ga0.6As quantum-dot lasers grown on Si substrates Appl. Phys. Lett. 74 $1355-7$

[81] Mi Z, Bhattacharya P, Yang J and Pipe K P 2005 Room-temperature self-organised In0.5Ga0.5As quantum dot laser on silicon Electron. Lett. 41742

[82] Wang T, Liu H, Lee A, Pozzi F and Seeds A 2011 13- $\mu \mathrm{m}$ InAs/GaAs quantum-dot lasers monolithically grown on Si substrates Opt. Express 1911381

[83] Lee A D, Qi Jiang, Mingchu Tang, Yunyan Zhang, Seeds A J and Huiyun Liu 2013 InAs/GaAs Quantum-Dot Lasers Monolithically Grown on Si, Ge, and Ge-on-Si Substrates IEEE J. Sel. Top. Quantum Electron. 19 1901107-1901107

[84] Tang M, Chen S, Wu J, Jiang Q, Dorogan V G, Benamara M, Mazur Y I, Salamo G J, Seeds A and Liu H 2014 13- $\mu$ m InAs/GaAs quantum-dot lasers monolithically grown on $\mathrm{Si}$ substrates using InAlAs/GaAs dislocation filter layers Opt. Express 2211528

[85] Chen S, Tang M, Wu J, Jiang Q, Dorogan V, Benamara M, Mazur Y, Salamo G, Seeds A J and Liu H 2014 1.3- $\mu \mathrm{m}$ InAs/GaAs quantum-dot laser monolithically grown on Si Substrates operating over 100 C Electron. Lett. 50 1467-8

[86] Orchard J, Shutts S, Sobiesierski A, Wu J, Tang M, Chen S, Jiang Q, Elliott S, Beanland R, Liu H, Smowton P and Mowbray D 2016 In situ annealing enhancement of the optical properties and laser device performance of InAs quantum dots grown on Si substrates Opt. Express 24 6196-202

[87] Tang M, Chen S, Wu J, Jiang Q, Kennedy K, Jurczak P, Liao M, Beanland R, Seeds A and Liu H 2016 Optimizations of Defect Filter Layers for 1.3- $\mu \mathrm{m}$ InAs/GaAs Quantum-Dot Lasers Monolithically Grown on Si Substrates IEEE J. Sel. Top. Quantum Electron. 22 50-6

[88] Chen S, Tang M, Wu J, Jiang Q, Dorogan V, Benamara M, Mazur Y, Salamo G and Liu H 2015 Long-Wavelength InAs/GaAs Quantum-Dot Light Emitting Sources Monolithically Grown on Si Substrate Photonics $2646-58$

[89] Tischler M A, Katsuyama T, El - Masry N A and Bedair S M 1985 Defect reduction in GaAs epitaxial layers using a GaAsP - InGaAs strained - layer superlattice Appl. Phys. Lett. 46294 $-6$

[90] Fischer R, Neuman D, Zabel H, Morkoç H, Choi C and Otsuka N 1986 Dislocation reduction in epitaxial GaAs on $\mathrm{Si}(100)$ Appl. Phys. Lett. 48 1223-5

[91] Tournié E, Cerutti L, Rodriguez J, Liu H and Wu J 2016 Metamorphic III-V semiconductor lasers grown on silicon MRS

[92] Volz K, Beyer A, Witte W, Ohlmann J, Németh I, Kunert B and Stolz W 2011 GaPnucleation on exact $\mathrm{Si}$ (001) substrates for III/V device integration J. Cryst. Growth 315 37-47

[93] Liu A, Peters J, Huang X, Jung D, Norman J, Lee M, Gossard A and Bowers J 2017 Electrically pumped continuous-wave $1.3 \mu \mathrm{m}$ quantum-dot lasers epitaxially grown on on-axis (001) GaP/Si Opt. Lett. 42338

[94] Huang X, Song Y, Masuda T, Jung D and Lee M 2014 InGaAs/GaAs quantum well lasers grown on exact $\mathrm{GaP} / \mathrm{Si}$ (001) Electron. Lett. 50 1226-7 
[95] Liu A, Zhang C, Snyder A, Lubyshev D, Fastenau J, Liu A, Gossard A and Bowers J 2014 MBE growth of P-doped $1.3 \mu \mathrm{m}$ InAs quantum dot lasers on silicon J. Vac. Sci. Technol. B, Nanotechnol. Microelectron. Mater. Process. Meas. Phenom. 32 02C108

[96] Jung D, Norman J, Kennedy M J, Shang C, Shin B, Wan Y, Gossard A C and Bowers J E 2017 High efficiency low threshold current $1.3 \mu \mathrm{m}$ InAs quantum dot lasers on on-axis (001) GaP/Si Appl. Phys. Lett. 111122107

[97] Yamaguchi M, Sugo M and Itoh Y 1989 Misfit stress dependence of dislocation density reduction in GaAs films on Si substrates grown by strained - layer superlattices Appl. Phys. Lett. $542568-70$

[98] Jung D, Zhang Z, Norman J, Herrick R, Kennedy M J, Patel P, Turnlund K, Jan C, Wan Y, Gossard A C and Bowers J E 2018 Highly Reliable Low-Threshold InAs Quantum Dot Lasers on On-Axis (001) Si with 87\% Injection Efficiency ACS Photonics 5 1094-100

[99] Norman J, Kennedy M, Selvidge J, Li Q, Wan Y, Liu A Y, Callahan P, Echlin M, Pollock T, Lau K M, Gossard A and Bowers J 2017 Electrically pumped continuous wave quantum dot lasers epitaxially grown on patterned, on-axis (001) Si Opt. Express 253927

[100] Li Q, Wan Y, Liu A Y, Gossard A C, Bowers J E, Hu E L and Lau K M 2016 13- $\mu$ m InAs quantum-dot micro-disk lasers on V-groove patterned and unpatterned (001) silicon Opt. Express 2421038

[101] Li Q, Ng K W and Lau K M 2015 Growing antiphase-domain-free GaAs thin films out of highly ordered planar nanowire arrays on exact (001) silicon Appl. Phys. Lett. 106072105

[102] Li J Z, Bai J, Major C, Carroll M, Lochtefeld A and Shellenbarger Z 2008 Defect reduction of GaAs/Si epitaxy by aspect ratio trapping J. Appl. Phys. 103106102

[103] Wan Y, Li Q, Geng Y, Shi B and Lau K M 2015 InAs/GaAs quantum dots on GaAs-on-Vgrooved-Si substrate with high optical quality in the $1.3 \mu \mathrm{m}$ band Appl. Phys. Lett. 107 081106

[104] Chen S, Liao M, Tang M, Wu J, Martin M, Baron T, Seeds A and Liu H 2017 Electrically pumped continuous-wave $13 \mu \mathrm{m}$ InAs/GaAs quantum dot lasers monolithically grown on onaxis $\mathrm{Si}(001)$ substrates Opt. Express 254632

[105] Uen W, Li Z, Huang Y, Chen M, Yang T, Lan S, Wu C, Hong H and Chi G 2006 Heteroepitaxial growth of GaAs on Si by MOVPE using a-GaAs/a-Si double-buffer layers J. Cryst. Growth 295 103-7

[106] Yu H, Chang E, Yamamoto Y, Tillack B, Wang W, Kuo C, Wong Y and Nguyen H 2011 Effect of graded-temperature arsenic prelayer on quality of GaAs on $\mathrm{Ge} / \mathrm{Si}$ substrates by metalorganic vapor phase epitaxy Appl. Phys. Lett. 99171908

[107] Zhou X, Pan J, Liang R, Wang J and Wang W 2014 Epitaxy of GaAs thin film with low defect density and smooth surface on Si substrate J. Semicond. 35073002

[108] Kwoen J, Jang B, Lee J, Kageyama T, Watanabe K and Arakawa Y 2018 All MBE grown InAs/GaAs quantum dot lasers on on-axis Si (001) Opt. Express 2611568

[109] Zhou Z P, Yin B and Michel J 2015 On-chip light sources for silicon photonics Light. Appl. 4 e358

[110] Palais J C 1988 Fiber optic communications (Prentice Hall Englewood Cliffs)

[111] Merckling C, Waldron N, Jiang S, Guo W, Collaert N, Caymax M, Vancoille E, Barla K, Thean A, Heyns M and Vandervorst W 2014 Heteroepitaxy of InP on Si(001) by selectivearea metal organic vapor-phase epitaxy in sub-50 nm width trenches: The role of the nucleation layer and the recess engineering J. Appl. Phys. 115023710

[112] Wang Z, Tian B, Pantouvaki M, Guo W, Absil P, Van Campenhout J, Merckling C and Van Thourhout D 2015 Room-temperature InP distributed feedback laser array directly grown on silicon Nat. Photonics 9 837-42

[113] Tian B, Wang Z, Pantouvaki M, Absil P, Van Campenhout J, Merckling C and Van Thourhout D 2017 Room Temperature O-band DFB Laser Array Directly Grown on (001) Silicon Nano Lett. 17 559-64

[114] Wang Y, Chen S, Yu Y, Zhou L, Liu L, Chuan C, Liao M, Tang M, Liu Z, Wu J, Li W, Ross I, Seeds A, Liu H and Yu S Monolithic Quantum-Dot Distributed Feedback Laser Array on Si Optica 5 
[115] Vahala K 2003 Optical microcavities Nature 424 839-46

[116] Maximov M V, Kryzhanovskaya N V, Nadtochiy A M, Moiseev E I, Shostak I I, Bogdanov A A, Sadrieva Z F, Zhukov A E, Lipovskii A A, Karpov D V, Laukkanen J and Tommila J 2014 Ultrasmall microdisk and microring lasers based on InAs/InGaAs/GaAs quantum dots Nanoscale Res. Lett. 9657

[117] Wan Y, Li Q, Liu A Y, Gossard A C, Bowers J E, Hu E L and Lau K M 2016 Optically pumped $13 \mu \mathrm{m}$ room-temperature InAs quantum-dot micro-disk lasers directly grown on (001) silicon Opt. Lett. 411664

[118] Wan Y, Li Q, Liu A Y, Chow W W, Gossard A C, Bowers J E, Hu E L and Lau K M 2016 Sub-wavelength InAs quantum dot micro-disk lasers epitaxially grown on exact $\mathrm{Si}(001)$ substrates Appl. Phys. Lett. 108221101

[119] Wan Y, Li Q, Liu A Y, Gossard A C, Bowers J E, Hu E L and Lau K M 2016 Temperature characteristics of epitaxially grown InAs quantum dot micro-disk lasers on silicon for on-chip light sources Appl. Phys. Lett. 109011104

[120] Shi B, Zhu S, Li Q, Tang C W, Wan Y, Hu E L and Lau K M $20171.55 \mu$ m roomtemperature lasing from subwavelength quantum-dot microdisks directly grown on (001) $\mathrm{Si}$ Appl. Phys. Lett. 110121109

[121] Shi B, Zhu S, Li Q, Wan Y, Hu E and Lau K 2017 Continuous-Wave Optically Pumped 1.55 $\mu \mathrm{m}$ InAs/InAlGaAs Quantum Dot Microdisk Lasers Epitaxially Grown on Silicon ACS Photonics 4 204-10

[122] Kryzhanovskaya N, Moiseev E, Polubavkina Y, Maximov M, Kulagina M, Troshkov S, Zadiranov Y, Guseva Y, Lipovskii A, Tang M, Liao M, Wu J, Chen S, Liu H and Zhukov A 2017 Heat-sink free CW operation of injection microdisk lasers grown on Si substrate with emission wavelength beyond $13 \mu \mathrm{m}$ Opt. Lett. 423319

[123] Wan Y, Norman J, Li Q, Kennedy M J, Liang D, Zhang C, Huang D, Zhang Z, Liu A Y, Torres A, Jung D, Gossard A C, Hu E L, Lau K M and Bowers J E 2017 1.3 $\mu$ m submilliamp threshold quantum dot micro-lasers on Si Optica 4940

[124] Wan Y, Jung D, Norman J, Shang C, MacFarlane I, Li Q, Kennedy M J, Gossard A C, Lau K $\mathrm{M}$ and Bowers J E 2017 O-band electrically injected quantum dot micro-ring lasers on on-axis (001) GaP/Si and V-groove Si Opt. Express 2526853

[125] Van der Ziel J, Dupuis R, Logan R and Pinzone C 1987 Degradation of GaAs lasers grown by metalorganic chemical vapor deposition on Si substrates Appl. Phys. Lett. 51 89-91

[126] Choi H, Wang C and Karam N 1991 GaAs - based diode lasers on Si with increased lifetime obtained by using strained InGaAs active layer Appl. Phys. Lett. 592634 - 5

[127] Kazi Z, Egawa T, Jimbo T and Umeno M 2000 First Room-Temperature Continuous-Wave Operation of Self-Formed InGaAs Quantum Dot-Like Laser on Si substrate Grown by Metalorganic Chemical Vapor Deposition Jpn. J. Appl. Phys. 39 3860-2

[128] Kazi Z, Thilakan P, Egawa T, Umeno M and Jimbo T 2001 Realization of GaAs/AlGaAs Lasers on Si Substrates Using Epitaxial Lateral Overgrowth by Metalorganic Chemical Vapor Deposition Jpn. J. Appl. Phys. 40 4903-6

[129] Groenert M, Pitera A, Ram R and Fitzgerald E 2003 Improved room-temperature continuous wave GaAs/AlGaAs and InGaAs/GaAs/AlGaAs lasers fabricated on Si substrates via relaxed graded Ge[sub x]Si[sub 1-x] buffer layers J. Vac. Sci. Technol. B Microelectron. Nanom. Struct. 211064 
Table:

Table I. Representative Summary of Reported Lifetime Data for GaAs Lasers on Si

\begin{tabular}{|c|c|c|c|c|c|c|}
\hline Year & Description & $\begin{array}{l}\text { Aging } \\
\text { Condition }\end{array}$ & $\mathrm{J}(0) / \mathrm{P}(0)$ & $\begin{array}{l}\text { Dislocation density } \\
\text { (method used) }\end{array}$ & Longest time to failure* & Ref. \\
\hline 1987 & GaAs/AlGaAs SQW & RT APC & $-/ 2 \mathrm{~mW}$ & $10^{7} \mathrm{~cm}^{-2}(-)$ & $<10 \mathrm{~s}$ & {$[125]$} \\
\hline 1991 & $\begin{array}{c}\mathrm{In}_{0.05} \mathrm{Ga}_{0.05} \mathrm{As} / \mathrm{AlGaAs} \\
\text { SQW }\end{array}$ & RT APC & $\begin{array}{l}\sim 2000 \mathrm{~A} / \mathrm{cm}^{-2} / \\
2 \mathrm{~mW}\end{array}$ & - & $10 \mathrm{~h}$ & {$[126]$} \\
\hline 2000 & $\begin{array}{c}\mathrm{In}_{0.2} \mathrm{Ga}_{0.8} \mathrm{As} \text { "QD like" } \\
\text { active region }\end{array}$ & RT APC & $\begin{array}{c}1320 \mathrm{~A} / \mathrm{cm}^{-2} / \\
0.5 \mathrm{~mW}\end{array}$ & - & $80 \mathrm{~h}^{* *}$ & [127] \\
\hline 2001 & $\begin{array}{c}\text { GaAs/AlGaAs SQW on } \\
\text { epitaxial lateral } \\
\text { overgrowth stripe }\end{array}$ & RT APC & $\begin{array}{l}810 \mathrm{~A} / \mathrm{cm}^{-2} / \\
1 \mathrm{~mW}\end{array}$ & $\begin{array}{c}2 \times 10^{6} \mathrm{~cm}^{-2} \text { (etch } \\
\text { pits) }\end{array}$ & $200 \mathrm{~h}$ & {$[128]$} \\
\hline 2003 & $\begin{array}{l}\mathrm{GaAs} / \mathrm{AlGaAs} \mathrm{SQW} \text { on } \\
\quad \text { graded } \mathrm{Ge}_{\mathrm{x}} \mathrm{Si}_{1-\mathrm{x}} / \mathrm{Si}\end{array}$ & RT ACC & $\begin{array}{l}270 \mathrm{~A} / \mathrm{cm}^{-2} / \\
<1 \mathrm{~mW}\end{array}$ & $\begin{array}{c}2 \times 10^{6} \mathrm{~cm}^{-2} \\
\text { (PVTEM + etch } \\
\text { pits ) }\end{array}$ & $4 \mathrm{~h}^{* *}$ & [129] \\
\hline 2014 & $\begin{array}{c}\text { InAs/GaAs QDs on } \\
\mathrm{Ge} / \mathrm{Si}\end{array}$ & $30^{\circ} \mathrm{C} \mathrm{ACC}$ & $\begin{array}{c}2000 \mathrm{~A} / \mathrm{cm}^{-2} / \\
16.6 \mathrm{~mW}\end{array}$ & $\begin{array}{l}2 \times 10^{8} \mathrm{~cm}^{-2} \\
(\mathrm{PVTEM})\end{array}$ & $\begin{array}{l}2100 \mathrm{~h} \text { (ageing test period) / } \\
4600 \mathrm{~h} \text { (extrapolated) }\end{array}$ & [79] \\
\hline 2016 & InAs/GaAs QDs on $\mathrm{Si}$ & $26^{\circ} \mathrm{C} \mathrm{ACC}$ & $\begin{array}{c}62.5 \mathrm{~A} / \mathrm{cm}^{-2} / \\
7.1 \mathrm{~mW}\end{array}$ & $\begin{array}{l}<10^{6} \mathrm{~cm}^{-2} \\
(\mathrm{PVTEM})\end{array}$ & $\begin{array}{l}3100 \mathrm{~h} \text { (ageing test period) / } \\
100158 \mathrm{~h} \text { (extrapolated) }\end{array}$ & [27] \\
\hline 2018 & $\begin{array}{c}\text { InAs/GaAs QDs on On- } \\
\text { Axis (001) Si }\end{array}$ & $35^{\circ} \mathrm{C} \mathrm{ACC}$ & - / $10 \mathrm{~mW}$ & $\begin{array}{l}8.4 \times 10^{6} \mathrm{~cm}^{-2} \\
\text { (ECCI) }\end{array}$ & $\begin{array}{c}1500 \mathrm{~h} \text { (ageing test period) / } \\
300140 \mathrm{~h} \text { (extrapolated) }\end{array}$ & [98] \\
\hline
\end{tabular}

TABLE II. Representative milestones of III-V/IV QD edge-emitting lasers by monolithic integration

\begin{tabular}{|c|c|c|c|c|c|c|}
\hline Year & $\lambda(\mathrm{nm})$ & Substrates & Operation condition & $\mathrm{J}_{\mathrm{th}}\left(\mathrm{A} / \mathrm{cm}^{-2}\right)$ & $\mathrm{T}_{\max }\left({ }^{\circ} \mathrm{C}\right)$ & Ref. \\
\hline 2005 & 1100 & $\mathrm{Si}(001)$ misoriented $4^{\circ}$ towards [111] & RT (Pulsed) & $\sim 1500$ & 95 & [81] \\
\hline 2011 & 1302 & $\mathrm{Si}(001)$ misoriented $4^{\circ}$ towards [110] & RT (Pulsed) & 725 & 42 & [82] \\
\hline 2011 & 1305 & $\mathrm{Ge}(001)$ misoriented $6^{\circ}$ towards [111] & $\mathrm{RT}(\mathrm{CW})$ & 55.2 & 60 & [76] \\
\hline 2012 & 1280 & $\mathrm{Ge} / \mathrm{Si}(001)$ misoriented $6^{\circ}$ towards [111] & RT (Pulsed / CW) & $64.3 / 163$ & $84 / 30$ & [77] \\
\hline 2014 & $\sim 1250$ & $\mathrm{Ge} / \mathrm{Si}(001)$ misoriented $6^{\circ}$ towards [111] & $\mathrm{RT}(\mathrm{CW})$ & $\sim 426$ & 119 & [78] \\
\hline 2016 & 1315 & $\mathrm{Si}(001)$ misoriented $4^{\circ}$ towards [110] & RT (Pulsed / CW) & $\sim 50 / 62.5$ & $120 / 75$ & [27] \\
\hline 2017 & 1280 & $\mathrm{GaP} / \mathrm{Si}(001)$ & $\mathrm{RT}(\mathrm{CW})$ & 860 & 90 & [93] \\
\hline 2017 & 1250 & V-groove Si (001) & $\mathrm{RT}(\mathrm{CW})$ & 333 & 80 & [99] \\
\hline 2017 & 1292 & $\mathrm{Si}(001)$ & RT (Pulsed / CW) & $240 / 425$ & $102 / 36$ & [104] \\
\hline 2018 & 1299 & $\mathrm{GaP} / \mathrm{Si}(001)$ & $\mathrm{RT}(\mathrm{CW})$ & 229.8 & 80 & [98] \\
\hline 2018 & 1250 & $\mathrm{Si}(001)$ & RT (Pulsed) & 320 & 70 & [108] \\
\hline
\end{tabular}

$\mathrm{RT}, \mathrm{CW}, \mathrm{J}_{\text {th }}$, and $\mathrm{T}_{\max }$ represent room temperature, continuous-wave, threshold current density, and the maximum lasing temperature, respectively. 
Figure Captions:

Figure 1: The monolithic integration issues: (a) APBs, (b) threading dislocations and (c) thermal cracks.

Figure 2: Schematic diagram of density states of bulk, quantum well and quantum dots materials.

Figure 3: The historical development of the record threshold current densities at the time of publication ( $\square$ QD laser on GaAs; $\square$ QD laser on Ge; $\bigcirc$ QD laser on SiGe; $\downarrow$ QD laser on Si.) CW indicates that the threshold current values were obtained from QD lasers under continuous operation. The rest were obtained from QD lasers tested in pulse mode. Reprinted by permission from IOP publishing [34], Copyright 2015

Figure 4: A comparison of conventional light-current (L-I) characteristics of QD (left) and QW (other two panels) Fabry-Perot (FP) lasers at different temperature. Reprinted by permission from Springer Nature: Nature Photonics [55], Copyright 2009.

Figure 5: (a) Schematic illustration of comparative interaction of threading dislocations with QDs and QWs. (b) Cross-sectional schematic description of the mechanism for dislocation bending by QD. (C) 2009 IEEE. Reprinted, with permission, from [69]. (c) Bright-field scanning TEM images of the threading dislocations in QD active region. Reprinted by permission from Springer Nature: Nature Photonics [27], Copyright 2016.

Figure 6: Comparison of room-temperature PL measurements for (a) single InAs QD layer and (b) single $8 \mathrm{~nm} \mathrm{In}_{0.20} \mathrm{Ga}_{0.80}$ As QW grown on GaAs versus Si substrates. The inset of (a) and (b) presents the bright-field cross-sectional TEM images for QD laser and QW laser grown on Si, respectively. Single facet L-I properties for the (c) InAs QD and (d) $\operatorname{In}_{0.20} \mathrm{Ga}_{0.80} \mathrm{As}$ QW lasers grown on $\mathrm{Si}$ substrates. Reprinted with permission from ref [73], Chinese Laser Press.

Figure 7: AFM images $\left(5 \times 5 \mathrm{~mm}^{2}\right)$ of the surface morphology of $\mathrm{GaAs}$ grown on Ge substrates with (a) As prelayer and (b) Ga prelayer techniques. TEM images of the interfacial region between the GaAs buffer layer and Ge substrate with (c) As prelayer and (d) Ga prelayer. (e) RT emission spectra obtained from the five-layer InAs/GaAs QD laser on Ge in accordance with driving currents below and above threshold. (f) Temperature-dependent light output-current characteristics for InAs/GaAs QD laser on Ge. Reprinted by permission from Springer Nature: Nature Photonics [76], Copyright 2011.

Figure 8: (a) The schematic diagram of an InAs/GaAs QD laser structure on a Ge-on-Si substrate. (b) TEM images of the interface between the GaAs buffer layer and the Ge buffer layer (above), and the Ge buffer layer and Si substrates (below). (c) Typical L-I characteristic for 3.5-mm-long QD laser on a Ge/Si substrate under pulsed mode. The inset of (c) presents the emission spectra of InAs/GaAs QD laser at different injection currents below and above threshold. Reprinted with permission from ref [77], The Optical Society (OSA). (d) CW L-I properties for a p-doped $993 \times 5 \mu \mathrm{m}^{2} \mathrm{HR} / \mathrm{SiN}$ laser with different stage temperature. Reprinted from [78], with the permission of AIP Publishing. (e) Aging 
data (at $30{ }^{\circ} \mathrm{C} / 100 \mathrm{~mA}$ ) for a p-doped $993 \times 5 \mu \mathrm{m}^{2} \mathrm{HR} / \mathrm{SiN}$ QD laser on $\mathrm{Si}$ stressed on a probe station. (C) 2015 IEEE. Reprinted, with permission, from [79]

Figure 9: High-resolution high angle annular dark field scanning TEM image of (a) a Si substrate with $4^{\circ}$ offcut and (b) the $6 \mathrm{~nm}$-thick AlAs nucleation layer between GaAs buffer layer and a Si substrate (c) Bright-field scanning TEM image of DFLs. (d) Dislocation density evaluated at different positions, as indicated in (c). (e) PL spectrum for a QD active region grown on Si. Inset: representative AFM image of an uncapped QD sample grown on Si. (f) Corrected high-angle annular dark-field scanning TEM images (false colour) of a single QD. Reprinted by permission from Springer Nature: Nature Photonics [27], Copyright 2016.

Figure 10: (a) Typical CW L-I-V curves for a $50 \mu \mathrm{m} \times 3,200 \mu \mathrm{m}$ InAs/GaAs QD laser grown on a Si substrate at $18{ }^{\circ} \mathrm{C}$. (b) Temperature-dependent L-I characteristics for InAs/GaAs QD laser on Si. (c) Histogram of threshold current density distribution for QD lasers on Si at RT under pulsed mode operation. (d) Output power and threshold current changes for the InAs/GaAs QD laser on Si at a constant heatsink temperature of $26^{\circ} \mathrm{C}$ and $\mathrm{CW}$ driving current of $210 \mathrm{~mA}$ as a function of aging time. Reprinted by permission from Springer Nature: Nature Photonics [27], Copyright 2016.

Figure 11: (a) A SEM image of the cross section for a cleaved narrow-ridge laser grown on GaP/Si. Note that the approximate position of the active region is presented as red line. Reprinted with permission from [93], The Optical Society. (b) Measurement of CW L-I-V and wall-plug-efficiency characteristics for a laser device $\left(2.5 \times 1079 \mu \mathrm{m}^{2}\right)$ at $20^{\circ} \mathrm{C}$. (c) Temperature-dependent L-I curves as a function of heat sink temperatures. Reprinted from [96], with the permission of AIP Publishing. (d) Variation of CW threshold current during the $1500 \mathrm{~h}$ aging test for two exemplary $1641 \mu \mathrm{m}$ long devices. Reprinted (adapted) with permission from [98]. Copyright 2017, American Chemical Society.

Figure 12: (a) CW L-I-V curves for a $9 \times 1200 \mu \mathrm{m}^{2}$ device. The inset of (a) shows SEM image of inplane GaAs nanowires array on V-grooved Si substrate. Reprinted from [103], with the permission of AIP Publishing. (b) The optical spectra above threshold shows that the ground state lasing around $1250 \mathrm{~nm}$ degenerates to multimode lasing from higher order transverse modes. (c) Temperaturedependent CW L-I properties for an $8 \times 1200 \mu \mathrm{m}^{2}$ device with polished facet and 95\% HR-coated facet. Reprinted with permission from ref [99], The Optical Society (OSA).

Figure 13: (a) $5 \times 5 \mu \mathrm{m}^{2}$ AFM image of a $400 \mathrm{~nm}$-thick GaAs grown on un-optimized $\mathrm{Si}$ (001): High density of APBs with RMS roughness of $1.6 \mathrm{~nm}$. (b) $2 \times 2 \mu \mathrm{m}^{2}$ AFM image of double-stepped $0.15^{\circ} \mathrm{Si}$ (001) substrate after optimized preparation (annealing under $\mathrm{H}_{2}$ ). (c) $5 \times 5 \mu \mathrm{m}^{2} \mathrm{AFM}$ image of an APBs-free $150 \mathrm{~nm}$-thick GaAs grown on optimized $0.15^{\circ} \mathrm{Si}(001)$ with RMS roughness of $0.8 \mathrm{~nm}$. Reprinted from [38], 2016; licensed under a Creative Commons Attribution (C) license (d) Conventional CW L-I-V plots of InAs/GaAs QD laser grown on GaAs/Si (001) at RT. The inset of (d) shows the lasing spectrum above threshold current density. (e) Single facet temperature-dependent L-I characteristics of an InAs/GaAs QD laser on GaAs/Si (001) substrate under pulsed mode operation. The inset of (e) presents the natural logarithm of current density as a function of temperature ranging from $16{ }^{\circ} \mathrm{C}$ to $102{ }^{\circ} \mathrm{C}$. Reprinted with permission from ref [104], The Optical Society (OSA).

Figure 14: (a) The high resolution tilted SEM image of the gratings with a $\lambda / 4$ phase shift in the middle after the first plasma etch in a previous test run. Note that the shaded area indicates the active region. Inset: the near 90-degree image of the grating area showing high quality etch without residue. Scale bar applies to both images. (b) The cutaway view of our DFB lasers on Si showing the layer 
structure and etched gratings (not to scale). (c) The L-I-V characteristic of a single $1 \mathrm{~mm}$ long DFB laser on Si. (d) The optical spectra of a DFB laser array with different grating periods around their maximum output power levels before saturation at RT. Resolution: $0.1 \mathrm{~nm}$. Reprinted with permission from ref [114], The Optical Society (OSA).

Figure 15: (a) Schematic diagram of layer structure QD micro-disk laser on Si. The SEM image (left) shows cross section of the structure. The TEM image (right) presents QD active region. (b) RT CW emission spectra measured at an injection current of $\sim 1.4 \times$ threshold for QD micro-disk lasers on $\mathrm{Si}$ with diameters of 14 and $30 \mu \mathrm{m}$. Dashed curve: PL spectrum of QD material (not in scale); inset: SEM image of the micro-disk laser with diameter of $30 \mu \mathrm{m}$. Reprinted with permission from ref [122], The Optical Society (OSA). 


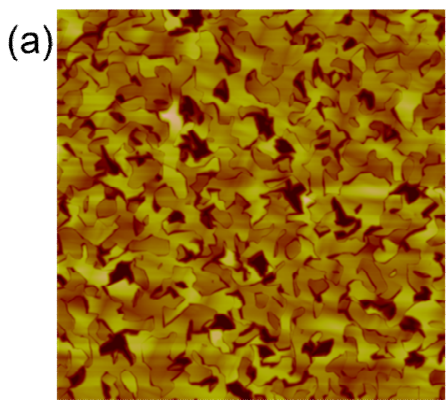

(b)

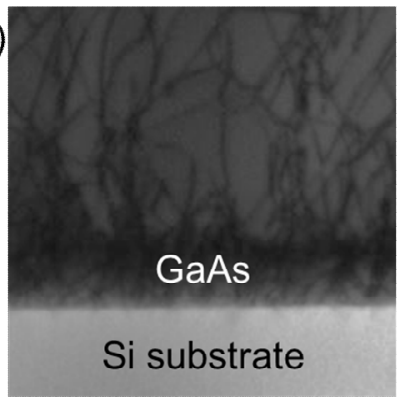

(c)

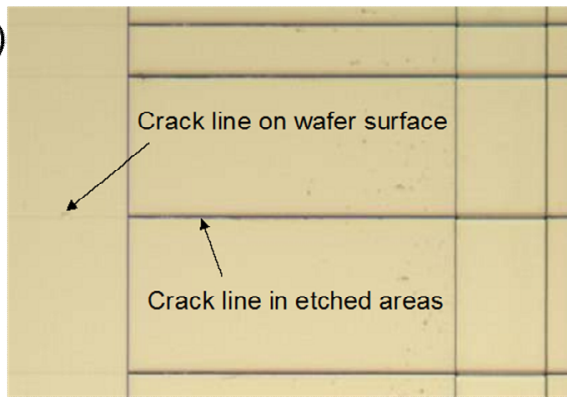

Figure 1 of 15
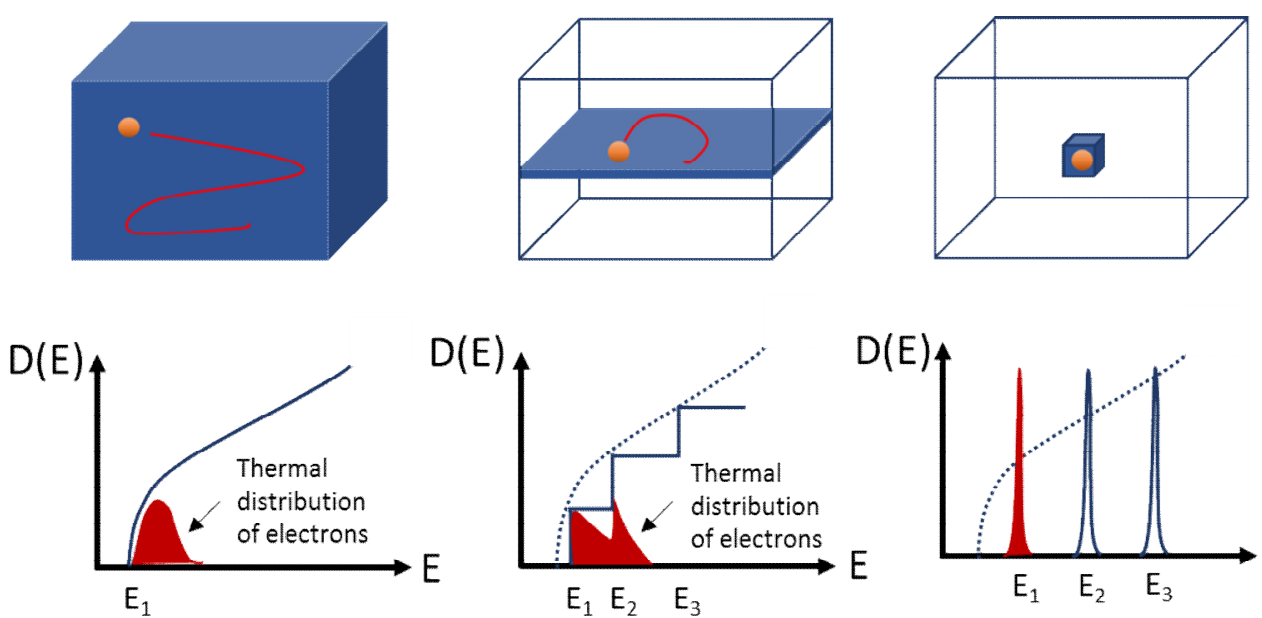

Bulk (3-D)
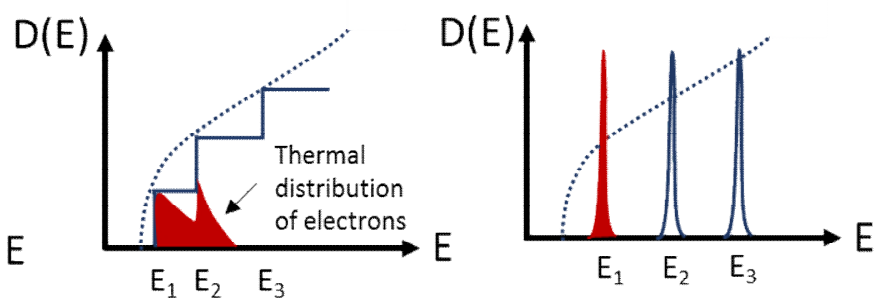

Quantum Well (2-D) Quantum Dot (O-D)

Figure 2 of 15 


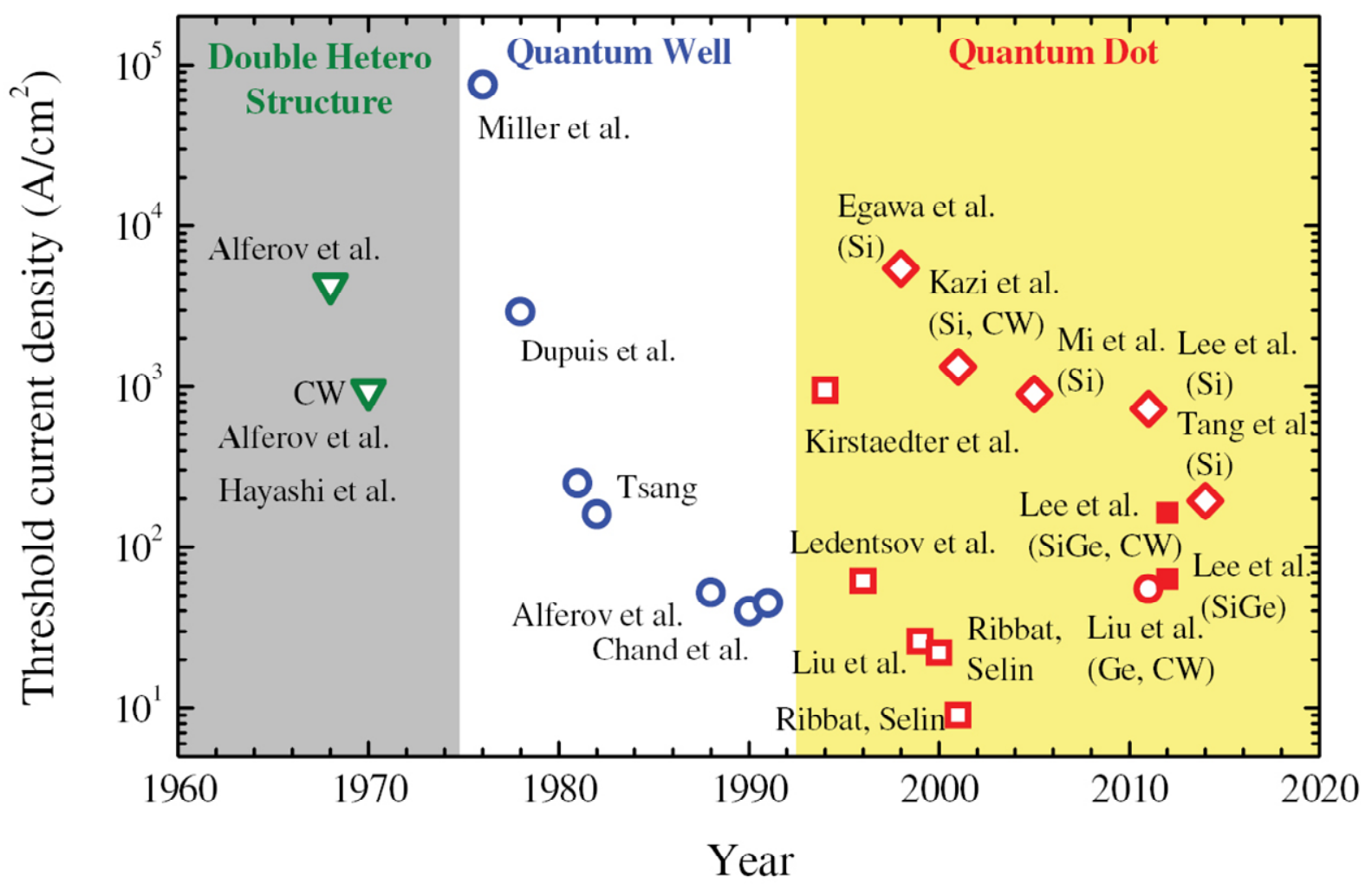

Figure 3 of 15

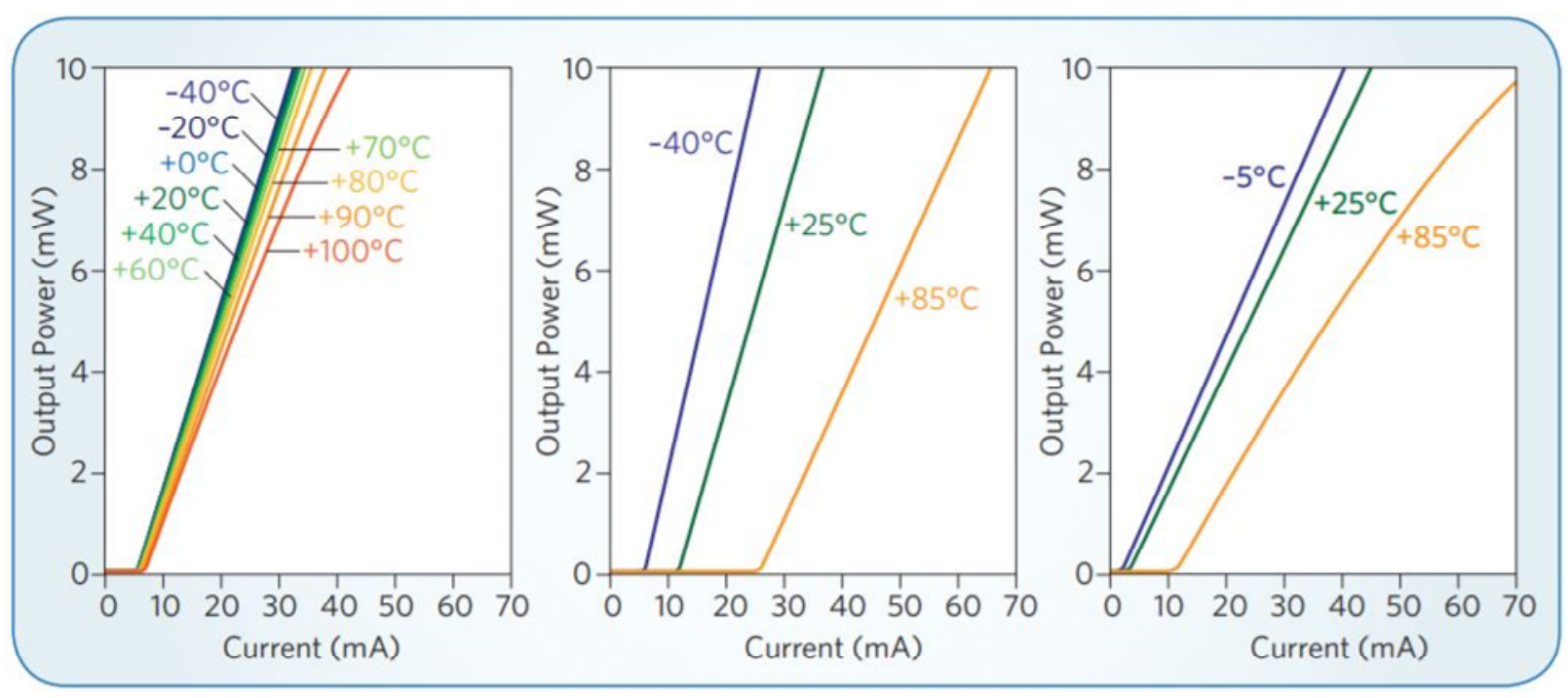

Figure 4 of 15 
(a) Threading dislocations

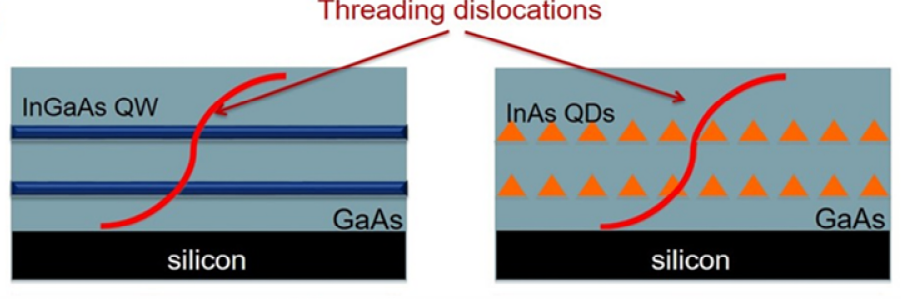

(b)

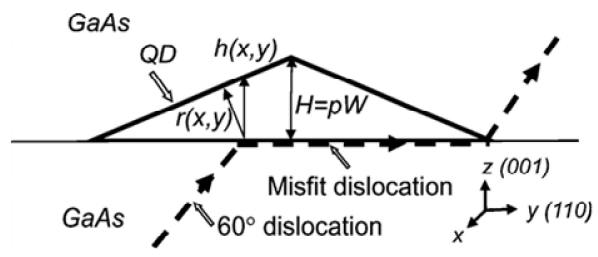

(c)

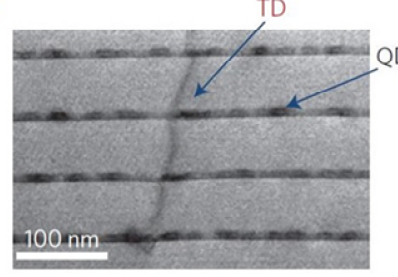

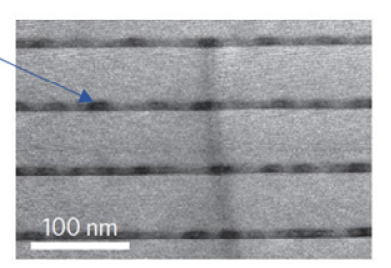

Figure 5 of 15
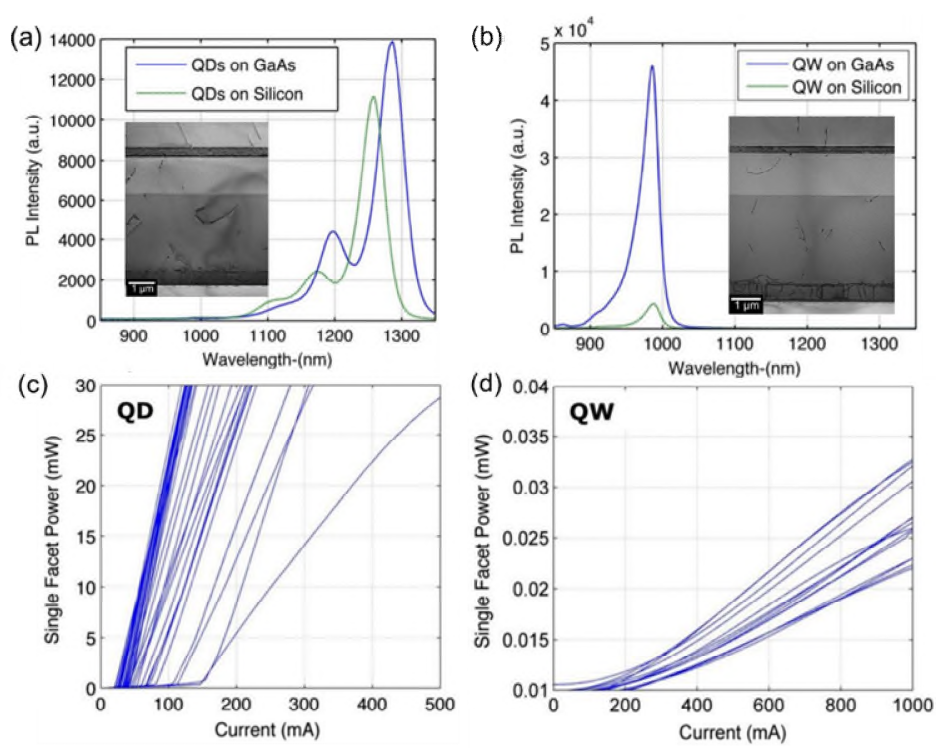

Figure 6 of 15
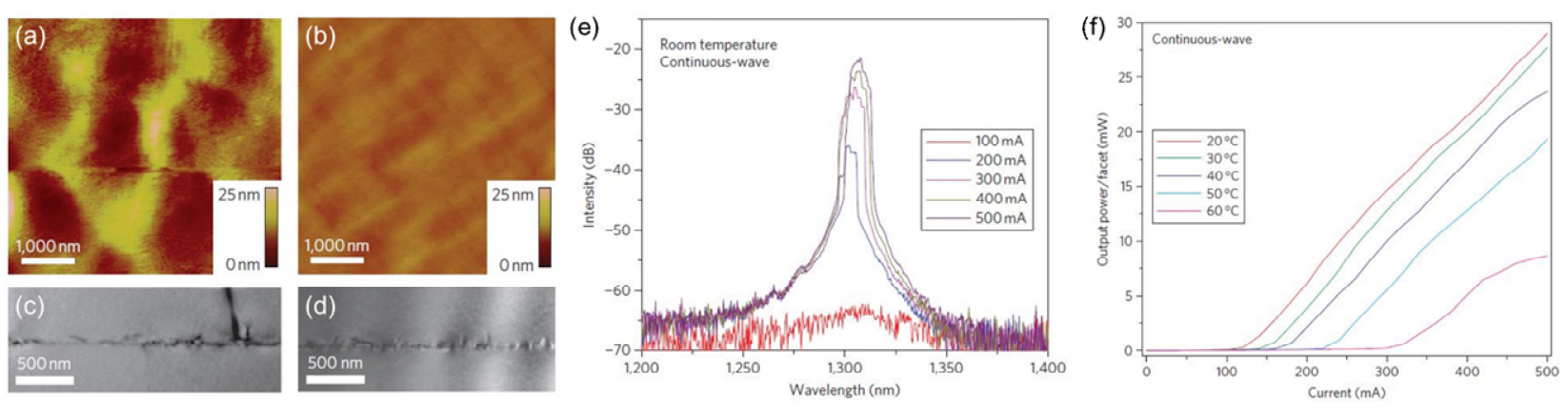

Figure 7 of 15 

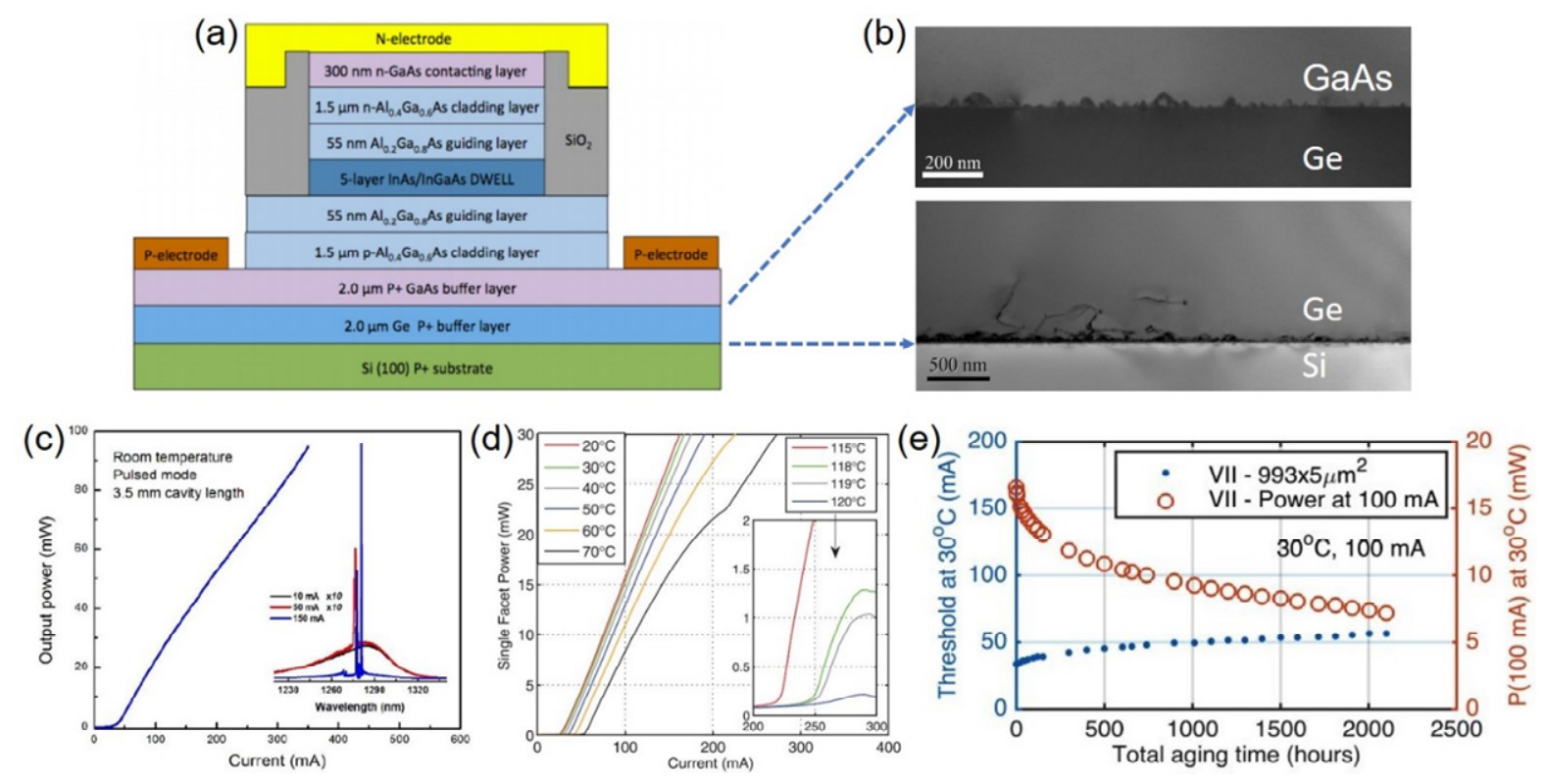

Figure 8 of 15
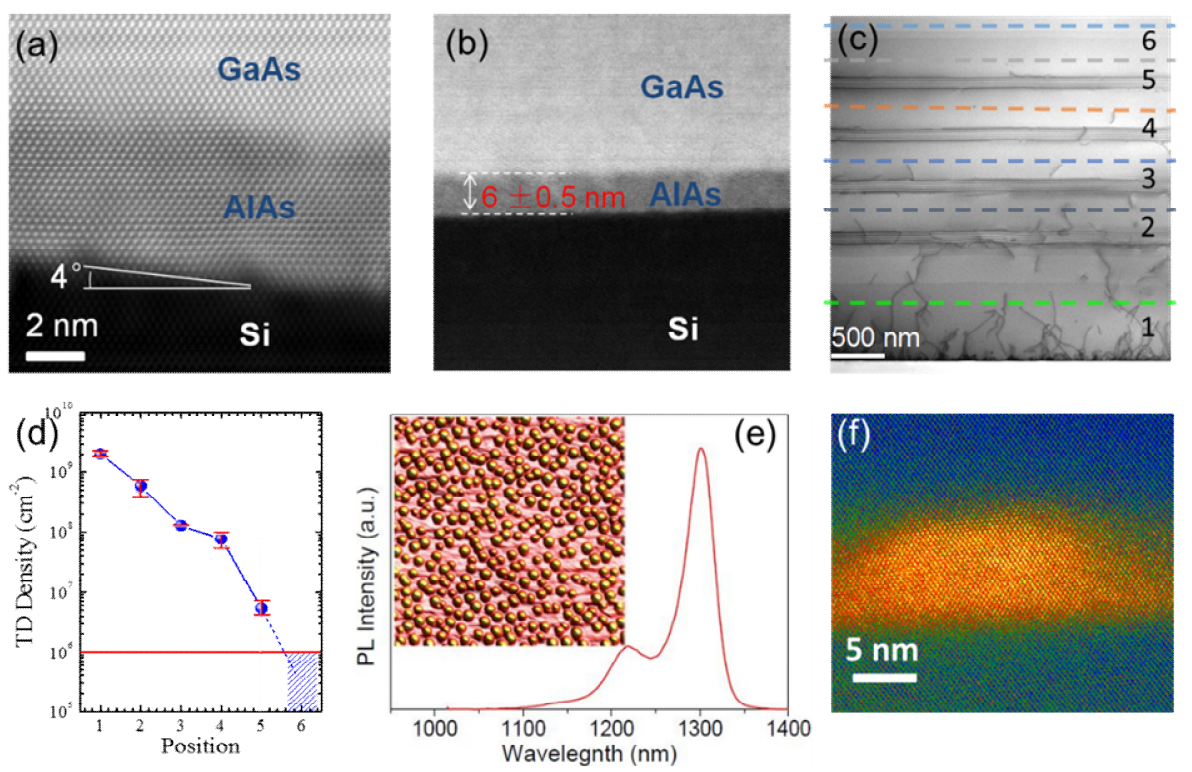

Figure 9 of 15 

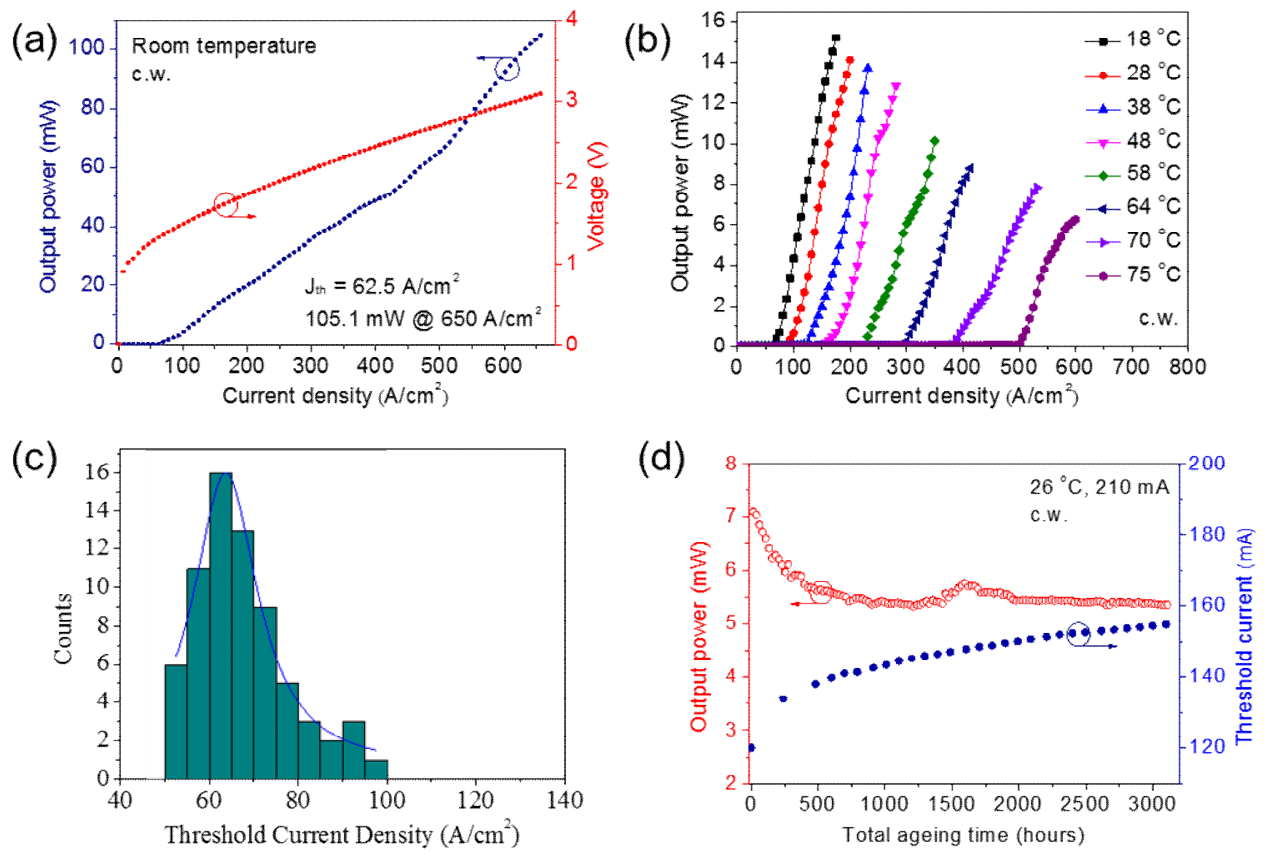

Figure 10 of 15
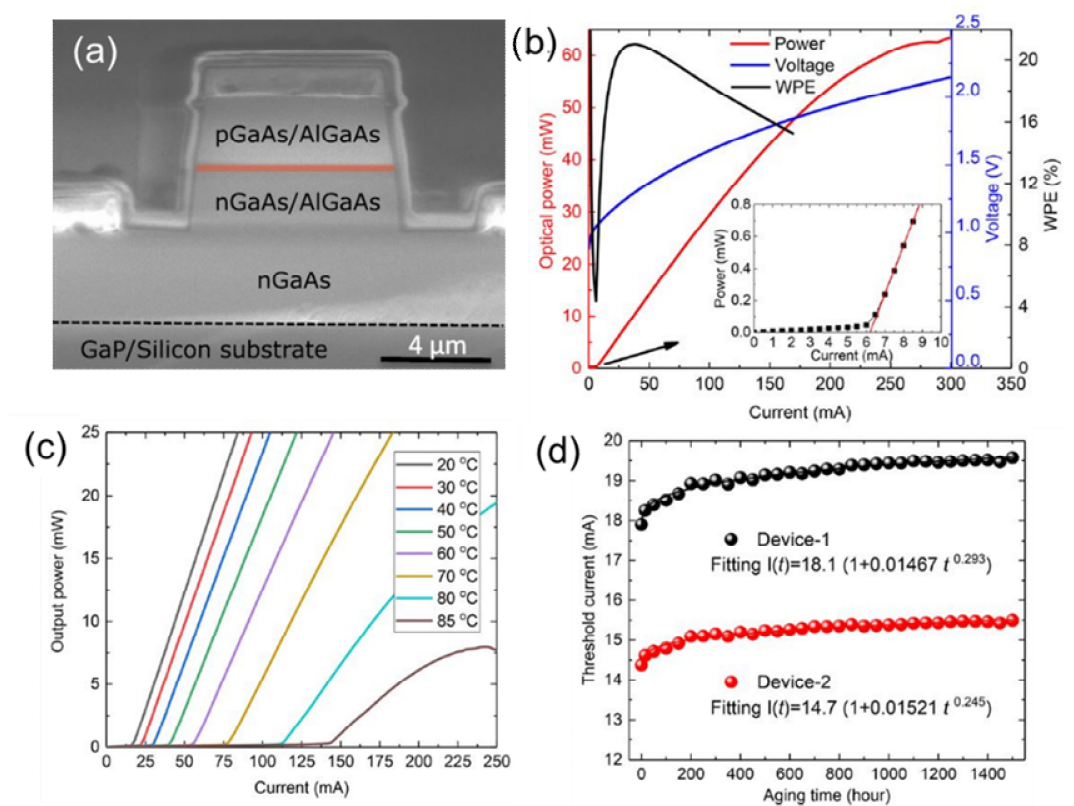

Figure 11 of 15

(a)

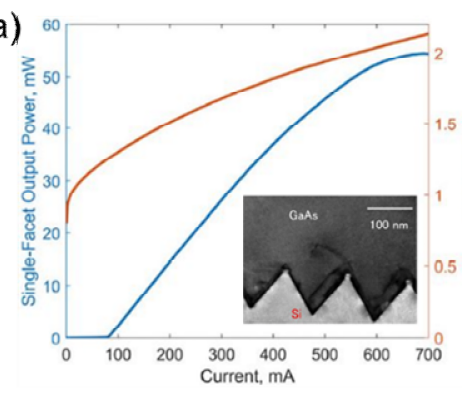

(b)

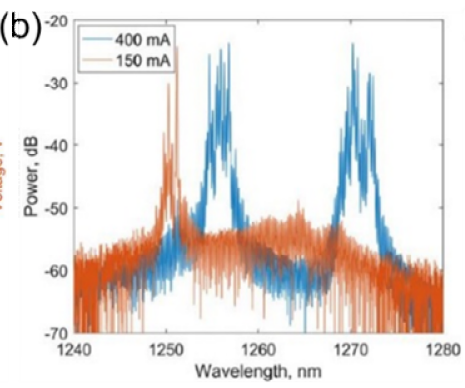

(c)

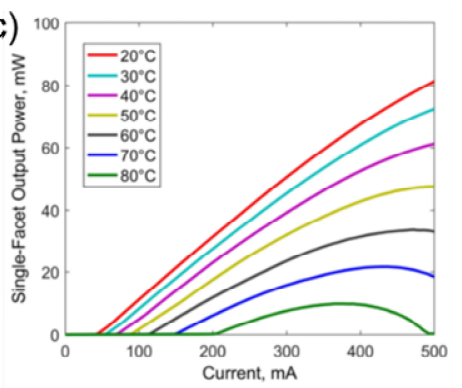

Figure 12 of 15 

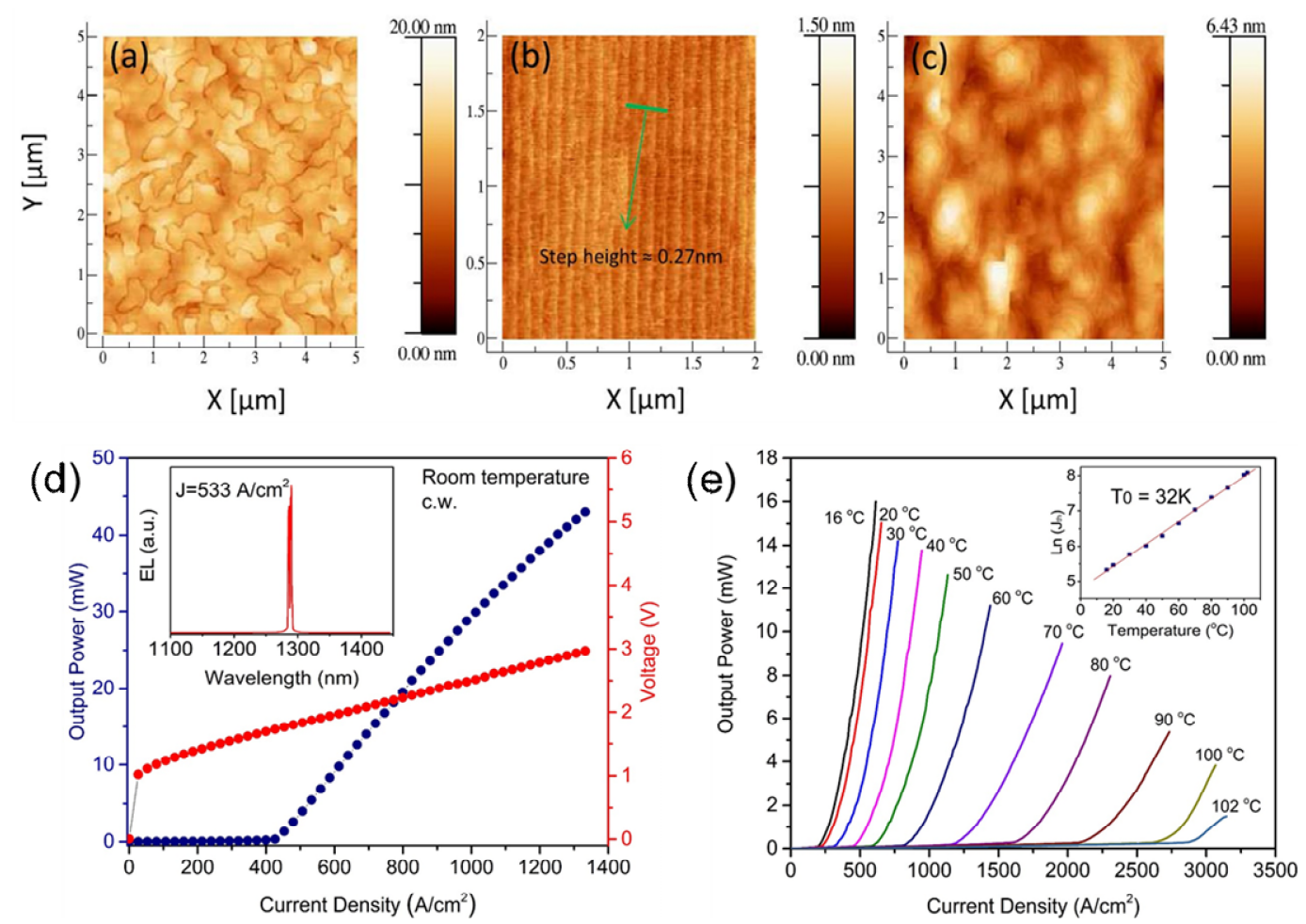

Figure 13 of 15
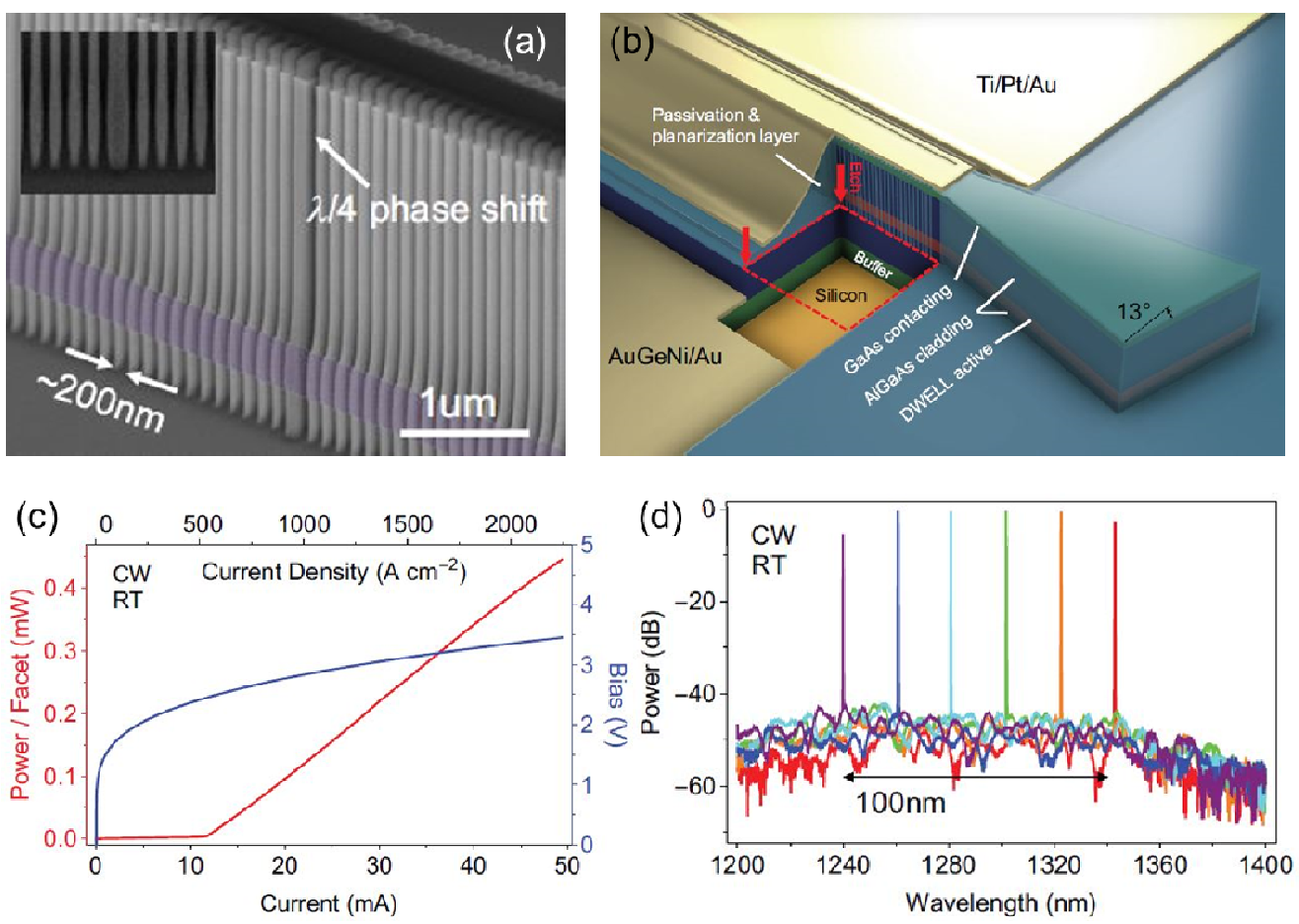

Figure 14 of 15 

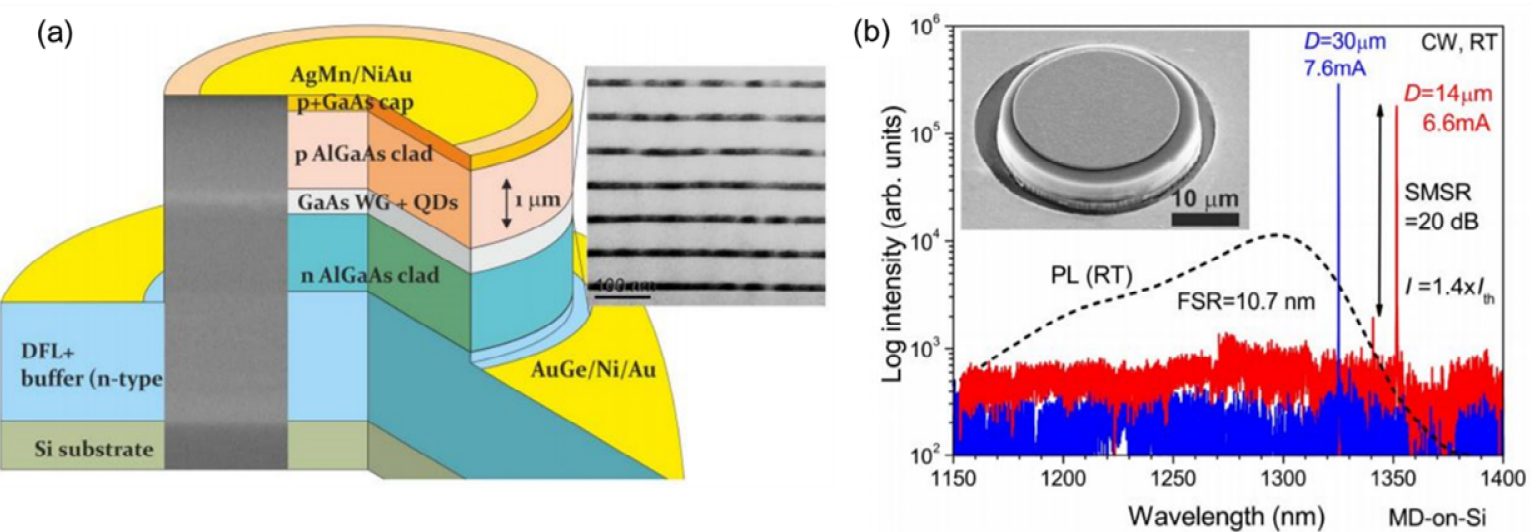

Figure 15 of 15 